\title{
Modeling and Simulation: Tools for Metabolic Engineering
}

Wolfgang Wiechert

Institute of Mechanical and Control Engineering,

Dept. of Simulation and Computer Science

Paul-Bonatz-Str. 9-11

University of Siegen, D-57068 Siegen, Germany

Phone: $-271-740-4727 \quad$ Fax: -2365

E-Mail: wiechert@simtec.mb.uni-siegen.de

To appear in: Journal of Biotechnology (2001) 
Abstract: Mathematical modeling is one of the key methodologies of metabolic engineering. Based on a given metabolic model different computational tools for the simulation, data evaluation, systems analysis, prediction, design and optimization of metabolic systems have been developed. The currently used metabolic modeling approaches can be subdivided into structural models, stoichiometric models, carbon flux models, stationary and nonstationary mechanistic models and models with gene regulation. However, the power of a model strongly depends on its basic modeling assumptions, the simplifications made and the data sources used. Model validation turns out to be particularly difficult for metabolic systems. The different modeling approaches are critically reviewed with respect to their potential and benefits for the metabolic engineering cycle. Several tools are discussed that have emerged from the different modeling approaches including structural pathway synthesis, stoichiometric pathway analysis, metabolic flux analysis, metabolic control analysis, optimization of regulatory architectures and the evaluation of rapid sampling experiments.

Keywords: Metabolic Engineering, Metabolic Modeling, Stoichiometry, Flux Analysis, Mechanistic Models, Metabolic Optimization, Model Validation 


\section{Introduction}

\section{Modeling for Metabolic Engineering}

The goal of metabolic engineering is the development of targeted methods to improve the metabolic capabilities of industrially relevant microorganisms. In order to reach this ambitious goal tools are required that assist in the evolutionary process of genetic manipulations of the cell metabolism and the improvement of bioprocess conditions. From an engineering perspective mathematical modeling is one of the most successful scientific tools available for this task. For technical systems the routine application of modeling and simulation software is already state of the art. In certain fields as for instance the design of analog electrical circuits, these tools have already reached a state of maturity that makes experiments and physical prototype development superfluous.

The challenge now is to transfer and adapt the developed modeling methodology from technical to biological systems. The question of the extent to which this is applicable remains open because there are several substantial differences between the two worlds as will be shown in the following. In particular, biological systems cannot be easily decomposed into unit components. However, our functional knowledge about cellular systems and the available database is growing dramatically. For this reason the present contribution surveys current progress in the modeling and analysis of metabolic networks. Emphasis is laid on the critical evaluation of model validity, on the classification of current models and methods, and on the application potential of model-based tools.

\section{Aims and Scope of Metabolic Models}

Starting with the modeling process for a given complex system the aim of the model should be specified and the task for which a model-based tool is intended to be developed. The following typical aims can be identified. They are ordered by an increasing demand for the precision and quality of the model: 
1. Structural understanding: Mathematical models are the most precise representation of knowledge because they have a unique and objective interpretation. Thus they do not permit any vague statements. Consequently modeling can be considered as a method to structure our often rather diffuse and entangled knowledge. Even if the model is not used for simulation this can already help to focus the attention on what is considered to be the essential parts of the system. In recent years this methodology has been extremely successful in the design of complex software systems (Rumbaugh et al., 1997).

2. Exploratory simulation: Undoubtedly the most frequent application of models is the exploration of the possible behavior of a system. Simulation scenarios based on rather crude mathematical models can help to achieve a rough understanding of the system behavior and to reject false hypotheses. Clearly, such speculative studies cannot directly help to produce the right ideas. Nevertheless, they help to focus on the most probable explanations for the behavior of the metabolic system. Many conceptual studies based on more or less simple models belong to this category. Several interesting examples are presented in (Heinrich and Schuster, 1996).

3. Interpretation and evaluation of measured data: The reproduction of experimental data by mathematical models is a well established tool in all scientific disciplines. For example, the characterization of growth, nutrient uptake and product formation by macrokinetic models has become a standard procedure in bioprocess development (Takors et al., 1997). However, it must be pointed out that in most cases this is merely a reproduction of the measured data. Thus it cannot be concluded that the respective model is a "good" one or even that it has any predictive power. Without further measures the fitting of models to data tends to produce a consistent "interpretation" rather than an "explanation" of the empirical results.

4. Systems analysis: Based on a given model mathematical methods can help to obtain a better understanding of the system's structure and its qualitative behavior. Of main interest are methods for the identification of functional units in metabolic and genetic systems, for the computation of stable states (Torres, 1994a), for the determination of parameter sensitivities (Albe and Wright, 1992; Torres, 1994b), for investigating the dynamic behavior (Shiraishi and Savageau, 1992), for explaining oscillating or even chaotic behavior (Goldbeter, 1996) or 
for computing theoretical limits of the systems metabolic capabilities (Edwards and Palsson, 1998). However, a more or less correct and precise model is required to obtain meaningful results.

5. Prediction and design: Based on a validated model the outcome of future experiments can be predicted. Clearly, the goal of this tool is the support of a rational design process for metabolic pathways. However, as will become clear later the validity and predictive power of metabolic models is often restricted to a narrow scope that does not always contain the intended target configuration.

6. Optimization: Once models are available that are predictive, at least in some parameter region, the ultimate goal of modeling in metabolic engineering can be tackled which is the computation of an optimal metabolic design (Hatzimanikatis et al., 1996). Such methods are state of the art in engineering disciplines like mechanics, electronics or civil engineering (Eschenauer et al., 1990). However, things are different with biotechnological systems.

Closely related to the model aim is the definition of the model scope and the required accuracy. The application of a model is always limited to a certain type of problem. For example, a stoichiometric network model (see below) is suitable for metabolic flux analysis but it contains no information about regulatory mechanisms. Thus it has little predictive power with respect to pathway alterations. Likewise model validation for regulatory models is usually done with measured data from a few physiological states (e.g. exponential growth in a batch culture). If a prediction is made outside this scope it must be treated with great care. Clearly, with respect to accuracy rough models can only produce rough predictions.

In general the following should be specified for a metabolic model: i) parameter and concentration range in which it is validated, ii) external conditions and inputs for which it works and iii) physiological modes for which it holds. As a rule, a model should always be as simple as possible and as complex as necessary. Clearly, the complexity of a model will grow if its scope and accuracy is extended. Thus the requirements should always be as modest as possible with respect to the problem to be solved. 


\section{Ingredients of a Metabolic Model}

For any mathematical model several types of modeling assumptions and the data sources used must be precisely documented in order to judge its applicability in a certain situation. The following aspects will later be discussed in more detail for each category of metabolic model:

1. Abstraction level: We are used to describing the complexity of cellular systems by different types of abstractions like gene maps, metabolic reaction networks, enzyme reaction mechanisms, geometric models, macromolecular structures and so on. Thus we focus on those cellular components and processes that are of interest in the current investigation and neglect the influence (and often even the presence) of all other parts. The basic abstraction of most metabolic models is the system compartmentalization into a number of homogeneously distributed pools connected by metabolic fluxes (Figure 1). The number of particles in each pool is so large that its stochastic fluctuations can be neglected. A concentration variable is then used to describe the state of the pool.

2. Modeling principles: Having chosen the level of abstraction the formulation of the model equations is guided by general modeling principles. In the case of compartmental modeling these are essentially the physical laws of mass and energy conservation. They lead directly to a set of balance equations that describe the system dynamics or equilibrium. Having determined the fluxes and pools that should belong to the model this is a strictly formal process and thus can be supported by modern modeling tools (Cellier, 1991).

3. Basic laws: One ingredient that is missing for the conversion of the balance equations into a structurally fully specified model is the representation of all fluxes in terms of the system state given by the vector of all metabolite concentrations. Usually the laws of enzyme kinetics as obtained from in vitro experiments (Cornish-Bowden and Wharton, 1988) are assumed also to hold in the intracellular environment.

4. Simplifying assumptions: Although a modeling framework might be capable of describing a metabolic system in considerable detail the number of equations and parameters arising are usually too large for a practical application of the model. For this reason simplifying assump- 
tions are made to reduce the model complexity. Frequently encountered assumptions are the lumping together of metabolite pools or the condensation of whole pathways into some few reaction steps (Vallino, 1991). If simplification is carefully done it seldom restricts the intended scope of the model.

5. Kinetic parameters used: When the kinetic laws are filled into the model it is almost ready for simulation. However, the kinetic constants are still missing. They are usually taken from the literature or available data-bases (Schomburg, 1997). Nevertheless, great care must be taken in this operation because the published constants were produced with different organisms and strains, different experimental and measurement methods and different evaluation approaches (Ewings and Doelle, 1980).

6. Measured data used: The most critical ingredient of the modeling process is measured data from the in vivo system. Clearly, complex metabolic models can only be validated with in vivo data from the functioning system. Different measurement techniques are under development to obtain a realistic picture of the functioning biological system. These are metabolic flux analysis (Vallino and Stephanopoulos, 1993), rapid sampling (Theobald et al. 1997), proteome analysis (Hatzimanikatis et al., 1999) or DNA chips (Derisi et al. 1997). They all produce enormous amounts of data that complement the parameter set taken from databases.

\section{Model Validation}

Only models that are valid within their declared scope lead to successful tools. Unfortunately, model validation is still the most difficult problem in the modeling process. It is already difficult to define what a valid model is in a precise mathematical sense. The common definition of validity is that a model can predict all experiments within the scope of the model. This is nothing else but a positive formulation of Popper's falsification principle (Popper, 1971): a model is valid if it resists all falsification attempts. The problem with this definition is that model validity can never be proven because there will always be one more experiment to do. Thus a more pragmatic approach is required from which an experimental validation procedure can be derived. Such con- 
cepts have been mainly developed in the statistical disciplines of regression analysis and experimental design.

A qualitative concept of model validation can be used if the precision requirements of the model are rather low. For many purposes it is then sufficient if the model is able to reproduce the basic behavior of the real system (e.g. stability, bifurcations, oscillations etc.) sufficiently well. This can be decided by methods from nonlinear systems theory.

A much stricter validation concept is derived from the falsification principle: if we cannot say what a good model is let's specify what a bad model is. Then try to find a model which explains the data and withstands all attempts to reveal it weaknesses. Clearly, this does still not guarantee a predictive model but it approximates this concept. The statistical procedure now is as follows: take a certain model and assume its validity, then fit the model parameters to the given data and apply a battery of statistical procedures to falsify the validity hypothesis. For example, all data must be sufficiently well reproduced and all predictions must have reasonably small confidence intervals (Seber and Wild, 1989).

An extension to this concept is model discrimination. In this case a family of differently structured models representing various possible explanation hypotheses for the function of the real system compete for the best explanation of the data set (Linhart and Zucchini, 1986). In general, the winner of this competition will be the "smallest" model that passes all statistical tests. Model discrimination can be further extended to a discriminating experimental design process in which further experiments are designed in order to discriminate between a family of models (Box and Hill, 1967). By this approach missing information for model validation is successively produced in a systematic way. It has been successfully applied to the discrimination of standard macrokinetic process models (Takors et al., 1997). 


\section{Promises of Metabolic Modeling}

It will become clear in the following that quite a lot of assumptions are required to build complex models of biological systems. While the validity of basic physical laws like mass conservation is indisputable and some biological assumptions like knowledge of the biochemical network are generally accepted, other assumptions like that of a constant $\mathrm{P} / \mathrm{O}$ ratio are highly speculative and not well justified. Thus the predictive power of some types of metabolic models is currently not very high.

It's worthwhile comparing this situation with the state of the art in the design of technical systems. Electrical circuits are a good example because they have much in common with chemical reaction networks. They are modeled on the basis of the abstraction of homogeneous electrical fields and the modeling principle is the conservation of charge so that electrical current balances resemble the metabolic flux balances (Cellier, 1991). The basic laws describe the behavior of resistors, capacities, inductivities etc. Simplifying assumptions are frequently made to describe active components like diodes or transistors or to neglect the effect of temperature.

The first big difference between electrical and metabolic systems is that in the electrical system any component can be isolated from the system without affecting its function. Secondly, the number of different component types (resistances, capacities, inductivities) in a circuit is quite small so that a few elementary laws can be reused in many places of the system. Thirdly, all possible interactions between the system components are exactly known because they are precisely given by the man-made circuit diagram. For these reasons it is state of the art in electrical circuit design to replace the experiment by a simulation without casting any doubt on the accuracy of the results.

Summarizing, the transfer of the modeling methodology from technical to biological processes is a very ambitious task and at the current state of knowledge it is unlikely that quantitatively predictive models with a broad scope will be obtained. It is to be expected that genome research will bring much greater functional knowledge about the biological system (Edwards and Palsson, 1998; Kao, 1999). Likewise in vivo measurement methods are under development that portray 
the intracellular state of a living cell as completely and as reliably as possible. The great challenge is to link these data sources in order to get the complete picture.

On the other hand, if less ambitious goals are set for metabolic modeling there are already a number of successful applications of modeling and system analysis. Some characteristic examples will be discussed in the next sections. The basic types of current modeling approaches in the field of metabolic engineering can be classified as indicated by the following sections. They are ordered by increasing mathematical complexity and requirement for model validation.

\section{Focus of the present review}

Mathematical models and simulation methods have been applied to cellular and metabolic systems for more than three decades and thus are not unique for metabolic engineering. The main focus of modeling in cell physiology always was the understanding of metabolic systems in the sense of the general principles which govern the cellular function. The new aspect of modeling in metabolic engineering is the usage of models for the targeted direction of metabolic fluxes in the sense of a rational engineering design.

Clearly, a thorough understanding of the complex metabolic regulation is always the best way to achieve such improvements. However it is in principle not necessary to aim at a deep understanding. Many successful technical applications of black box models like for example linear models or neural networks have proven the opposite. On the other hand models should always incorporate as much secured knowledge about the biological system as possible while wrong or uncertain assumptions might cause a loss of predictive power. Thus a major concern of the present review is the critical discussion of model validity.

It is not surprising that there are several methodological differences between the „older“ cell physiology community and the „younger“ metabolic engineering community. Only in recent years a scientific interchange between both communities has begun and in particular the terminology and the methods are translated if not unified. Interestingly this is no "one-way" relation 
because the system design aspect has also consequences for the system understanding aspect (Hofmeyer et al., 2000).

There are several excellent general introductions to metabolic engineering and certain of its aspects (Bailey, 1991; Stephanopoulos, Sinskey, 1993; Nielsen, 1998; Stephanopoulos, 1999). Likewise there are two excellent textbooks from the cellular physiology viewpoint at the one hand (Schuster, 1996) and the metabolic engineering viewpoint at the other hand (Stephanopoulos et al., 1998). Both also cover mathematical tools. The new aspects of the present review now is

- to develop a systematic framework by which current modeling activities can be classified and judged,

- to bring together and compare results from cell physiology and metabolic engineering,

- to thoroughly discuss the aspect of model validity which is a crucial aspect for the application of any model, and

- to emphasize the metabolic design aspect which sheds another light on the applicability of classical tools from cell physiology.

The classification hopefully is a helpful guide for the reader into a vast amount of literature, although it cannot cover any published modeling method. The review concentrates on publications with a major focus on modeling and mathematical tools. Because this is a text about metabolic engineering it primarily concentrates on the first decade of this discipline since about 1990. To keep the number of citations reasonably low standard textbooks are preferred over the explicit citation of articles from the seventies and eighties. 


\section{Structural Network Models}

\section{Basic assumptions}

The biochemical structure of at least the central metabolic pathways is the only biological knowledge whose validity is (almost) undisputed. All chemical reaction steps are known, the reaction mechanisms are well understood in most cases and also many cofactors and effectors are available from textbooks and databases (Michal, 1999; Kanehisa, 1999). On this basis, structural models of metabolism can be constructed which - in mathematical terminology - are represented by bipartite directed graphs (Figure 2). This means that there are two types of nodes, i.e. flux nodes and substance nodes. A flux node can only be connected to substance nodes and vice versa. The role of the substances in a reaction step (e.g. as a substrate, product, cofactor or inhibitor) can be further specified by annotating the arcs of the graph. There is no further need for parameters and measurement data.

\section{Developed tools}

Inspired by the graphical representation, methods from graph theory, network theory, automata theory or formal language theory can be applied. Typical results that can be achieved by these methods are:

1. Pathway modeling: The first step to structural analysis must be the specification of the network in some user-friendly way. Different textual and graphical tools have been developed to generate a structural network description from the user input (Hofestädt, 1993).

2. Pathway synthesis: Physiologically meaningful biochemical pathways can be computed by graph component analysis (Seressiotis and Bailey, 1988; Mavrovouniotis, 1992). They represent basic metabolic operation modes with a certain function. Likewise the discovery of regulatory loops and signaling cascades is possible (Kohn and Lemieux, 1991).

3. Qualitative dynamic analysis: The network structure can also be interpreted by a Petri net. Petri net tools can then be applied to analyze the network consistency and completeness in 
terms of deadlocks or unreachable states (Reddy et al., 1993). Another dynamic description is given by automata (Thomas, 1991).

4. Visualization tools: Flexible graphical network representations are urgently required to facilitate the interactive inspection of network structures and the visualization of all the quantitative results produced by the subsequently described tools. However, the problem of automatic graph drawing from a structural input is not that easy (Karp and Paley, 1994).

5. Optimal pathway synthesis: An application of structural methods that might be of interest in the metabolic design of new pathways is the computation of optimal reaction networks which produce certain product metabolites from given substrates. For example, the pentose phosphate pathway produces $\mathrm{C} 3$ - and $\mathrm{C} 4$-molecules from $\mathrm{C} 5$-molecules without waste. This task can be formulated as a combinatorial puzzle where generalized biochemical reaction steps (e.g. group transfer reactions) have to be combined to reach the goal. Then the reaction architecture is computed with a minimal number of reaction steps under certain thermodynamic feasibility constraints. In the case of the pentose phosphate pathway it was shown that the evolutionary solution to this problem is really optimal (Melendez-Hevia, 1994). However, if this method is applied to new design problems it remains unclear whether a suggested pathway constructed from generalized reaction steps is physically realizable by genetic engineering.

6. Functional genomics: While the discrete structure of the central metabolic pathways is rather well understood our knowledge about the whole metabolism and about the genetic regulation of enzyme expression is rather incomplete. In these areas the first goal must be to develop the structural model (Collado-Vides, J., 1989; Hatzimanikatis et al., 1999). The challenge of functional genomics is the unraveling of the regulatory network from known facts about gene expression patterns that e.g. are produced with DNA chips and electrophoresis gels (Kao, 1999).

7. Thermodynamic and qualitative reasoning: $\Delta \mathrm{G}^{0}$ constants must be treated with care because they are not in general valid for the nonequilibrium situation (Westerhoff and van Dam, 1987). Consequently, $\Delta \mathrm{G}^{0 \text { ' }}$ values may serve as a rather qualitative measure which imposes 
further constraints on the model. Some qualitative tools which take thermodynamic or other qualitative information into account are described in (Mavrovouniotis, 1993). The relation between thermodynamics and kinetics is discussed in (Nielsen, 1997).

\section{Stoichiometric Network Models}

\section{Basic assumptions and measured data}

Structural models do not contain any quantitative information about substance concentrations or metabolic fluxes. Thus structural methods can only detect possible regulatory structures but it remains unanswered whether these regulation mechanisms are also quantitatively relevant in a certain physiological state of the cells. In particular, if an enzyme is influenced by several antagonistic cofactors and effectors in a nonlinear way (like e.g. phosphofructokinase) almost anything can happen.

Most industrial production processes are operated under quasi-stationary conditions which means that the main process parameters, i.e. substrate concentration, oxygen concentration, dilution rate etc., are kept constant at least within time spans of about one hour. Because the regulatory time constants of metabolic systems are much smaller it is usually assumed that stationary conditions will also emerge in all metabolite pools of each single microbial cell. However, this is not necessarily the case. Oscillations are quite frequent in biological systems (Goldbeter, 1996) and have been observed in synchronized cultures. But the single cells may also fluctuate around a certain state because there is a cell cycle. Then the overall impression of the cell population would be that of a stationary system because the cell cycles are not synchronized. It is currently not known how large this effect is. But even if each single cell had quasi-stationary pool sizes it might be the case that different subpopulations have different flux patterns. Unfortunately, the development of experimental single cell methods is only at the beginning and thus no data is available to quantitate these effects. Summarizing, the assumption of quasi-stationarity is well justified for the time average of a structured cell population. On the other hand, fluxes observed in a cell population 
cannot in general be transferred to a single cell. Thus - if required - it must be postulated that this transfer is possible.

If constant fluxes and intracellular pool sizes are assumed for the "average" cell the stoichiometry of the metabolic network induces a set of linear relationships between the metabolic fluxes (Figure 2) which is generally expressed as

$$
\mathbf{N} \cdot \mathbf{v}=\mathbf{0}
$$

where $\mathbf{N}$ is the stoichiometric matrix and $\mathbf{v}$ is the vector of all metabolic fluxes. The stoichiometric relations can be written for net fluxes as well as for separated forward and backward fluxes (cf. Figure 5). The latter becomes necessary if both directions of bidirectional reactions steps need to be formally considered as different reactions. An important difference between net fluxes and forward/backward fluxes is that the latter must always be positive while the net flux as the difference of forward and backward flux - can have both signs.

A crucial simplification necessary for compartmental modeling in practice is the lumping of pools. Usually, pools which are connected by rapidly exchanging reactions (e.g. the ribose-5-, ribulose-5- and xylulose-5-phosphate pool in the pentose phosphate pathway) are lumped together into one single pool. Similarly, linear reaction sequences without any branch point (e.g. the oxidative pentose phosphate pathway) can be lumped into a single reaction step (Vallino, 1991). However it must be taken care that only so called monofunctional units are considered as a module (Rohwer et al., 1996).

Moreover, there are many reaction steps in complex metabolic models which are not modeled in detail because they are assumed to be of minor importance. For example, all the anabolic reactions outside the central metabolism cannot be modeled in detail (i.e. for each single step) because this would expand the model to an unmanageable size. Thus they are lumped together into formal reaction steps. Non-integer stoichiometric constants may occur in this case because formal pools like "cell mass", "energy supply" or "organic acids" may be contained. A particularly important fact is that biomass is always composed of 12 elementary precursor metabolites (Neidhardt et al., 1990). The percentual precursor demand to synthetize the cell mass is assumed to be constant under quite varying nutritional conditions (Vallino, 1991). This produces a very important set of 12 noninteger coefficients. 
A special problem is the intracellular compartmentalization of eucaryotes where reaction steps are localized in a specific compartment. For example, the citric acid cycle takes place inside the mitochondria. On the other hand there are some reactions that can be found in several compartments and thus must be duplicated to obtain a correct network model. For example there are two functioning glycolytic pathways in plant cells (Rees, 1988). The different compartments are linked by transport steps over the intracellular membranes which have to be incorporated into the flux model.

One of the most critical assumptions in stoichiometric modeling is the balancing of the "energy metabolites" ATP, UTP, NADH, NADPH etc. To this end, the stoichiometric coefficients of some very complex intracellular processes must be assumed. Firstly, the generation of ATP from $\mathrm{NADH}$ and oxygen must be described by a $\mathrm{P} / \mathrm{O}$ ratio which is usually around 2.5. However, there is a continuing discussion about this ratio and its dependence on intracellular conditions (Brand, 1994; Fitton et al. 1994; Sauer and Bailey, 1999). Secondly, the ATP requirement for biomass synthesis must be assumed. Thirdly, all processes producing and consuming the energy metabolites must be known quantitatively. This makes energy balancing a rather doubtful procedure and indeed flux analysis studies that do not rely on energy balancing (see below) showed that the energy balances are not closed (Marx et al., 1996).

Several metabolic fluxes can be directly measured. These are the "extracellular" fluxes like product formation, oxygen uptake or carbon dioxide evolution and the growth rate. Additionally when a detailed chemical analysis of cell mass composition is available - the corresponding precursor demands can be directly computed from the growth rate (Vallino, 1991). This produces another set of directly measured metabolic fluxes.

\section{Developed tools}

Having accepted the mentioned assumptions and simplifications, the stoichiometric model can be used for different purposes. The key to all these tools is that the space of all possible flux patterns $\mathbf{v}$ is strongly restricted by the stoichiometric relations to a low-dimensional linear subspace 
(Schilling et al., 1999). In addition to the measurable precursor effluxes there typically remain about 5 degrees of freedom for the central metabolic pathways of a procaryotic cell. The exploitation of this linear flux space led to the development of some powerful tools:

1. Matrix generation: The key to all stoichiometric methods is the automatic generation of the stoichiometric matrix $\mathbf{N}$ from a textual input in a familiar chemical reaction notation. Several modeling tools have been developed (Vallino, 1991; Pfeiffer et al., 1999). Once the matrix has been generated, subsequent methods are based on standard matrix calculations as are readily available within scientific computing environments like MATLAB or MATHEMATICA.

2. Metabolic flux analysis: Stoichiometry-based MFA complements the stoichiometric relations by the measured fluxes. If the flux measurements are nonredundant and if not too many degrees of freedom remain all intracellular metabolic fluxes can be estimated from the data (Vallino and Stephanopoulos, 1993; Varma, Palsson, 1994). This turns out to be a classical linear estimation exercise for which all relevant problems have been solved (Vallino, 1991; Van Heijden et al., 1994a,b): the structural identifiability of the fluxes can be decided, all fluxes can be efficiently computed, the available redundant information is fully used, a confidence region for the estimated fluxes can be computed, the set of redundancy relations for the measured data can be derived explicitly, and gross measurement errors can be detected. Thus from a methodological viewpoint stoichiometry-based metabolic flux analysis is a mature tool for metabolic engineering. However, without energy balancing the flux balances are usually underdetermined.

3. Extreme flux patterns: Forward and backward fluxes are now considered separately in the stoichiometric relations. Additionally some fluxes can be assumed to be unidirectional based on thermodynamic considerations, i.e. the back flux is set to zero. If the substrate uptake is scaled to one (i.e. $100 \%$ ) the arising inequality $\mathbf{v} \geq \mathbf{0}$ then further restricts the set of possible flux patterns to a convex polyhedron in the flux space (Figure 3) (Clarke, 1988). Any point in this polyhedron represents a feasible flux pattern of the system. The corner points of the polyhedron - called the extreme points or the extreme flux patterns - are of special interest 
because they exactly determine the feasible flux space. Algorithms for the calculation of extreme points are available (Hohenbalken et al., 1987).

An extreme flux pattern is characterized by the fact that the number of fluxes which vanish in this flux pattern is at a local maximum. Thus the extreme flux patterns can be interpreted as basic metabolic operation modes where only some of the reaction steps are active (Figure 4). Any other flux pattern can be represented by a weighted combination of the extreme flux patterns. This gives an understanding of the metabolic network in terms of certain distinctive physiologically patterns.

Optimal flux patterns: A rather speculative extension to the extreme point analysis is the complementation of the stoichiometric relations by a linear metabolic optimization criterion. Several criteria like maximal growth rate, maximal product formation or a minimal ATP production for a given substrate uptake have been investigated (Edwards and Palsson, 1998). They all lead to a classical linear programming problem which can be solved by the simplex algorithm. Optimality criteria have also been used to solve the metabolic flux balances in the case where the measured data (even with energy balancing) is still not sufficient to compute a unique solution (Bonarius et al., 1997).

Except for degenerate cases the optimization result is always an extreme flux pattern. However, such a result need not represent a reasonable solution. For example the maximal lycine production rate for C. glutamicum has been computed to more than $60 \%$ of the glucose uptake rate in (Vallino, 1991) while current values from production processes are below $30 \%$. The difference comes from the fact that in order to reach maximal product formation the organism must stop any "waste" of energy for growth or maintenance.

4. Elementary flux modes: Extreme flux patterns are not always convincing solutions to the problem of finding physiologically meaningful pathways in a metabolic network. Several approaches have been undertaken to obtain a biologically meaningful and yet mathematically precise definition of a metabolic pathway. A recent definition of such elementary flux modes requires that an elementary mode has a maximal number of vanishing fluxes and cannot be decomposed into smaller pathways (Schuster et al., 1999). Thus elementary modes are the 
smallest functioning subunits of a metabolic network. This motivates the hypothesis that they are also genetically regulated as a unit which in turn is a promising approach to the development of functional genomics tools. Elementary modes can be efficiently calculated by a newly designed algorithm that has some similarity to the simplex algorithm (Pfeiffer et al., 1999). They have several promising applications in metabolic design, drug development or functional genomics (Schuster et al., 1999).

\section{Carbon Flux Models}

\section{Basic assumptions and measured data}

Stoichiometry-based flux analysis suffers from the disadvantage that the energy metabolites must be balanced in detail, parallel pathways cannot be distinguished, certain cycle fluxes are not observable and bidirectional steps cannot be resolved (Wiechert, 2001). Otherwise there is not enough measurement information to fix the degrees of freedom left by the stoichiometric relations (Bonarius, 1997). Thus the corresponding flux analysis results must always be used with great care. This gave rise to an extension of the measured data set by ${ }^{13} \mathrm{C}$ carbon labeling measurements.

A carbon labeling experiment is carried out by feeding a specifically ${ }^{13} \mathrm{C}$-labeled substrate in a metabolic stationary state (Wiechert and de Graaf, 1996). Frequently used substrate molecules are $1-{ }^{13} \mathrm{C}$ glucose or uniformly labeled glucose. The ${ }^{13} \mathrm{C}$ isotopes are then distributed over the whole metabolic network until finally an isotopically stationary state emerges in which all percentages of the differently labeled molecules in each metabolite pool become constant. In this state labeling measurements are taken by using ${ }^{1} \mathrm{H}-\mathrm{NMR},{ }^{13} \mathrm{C}-\mathrm{NMR}$ or MS instruments. Details of the experimental procedures and a comparison of the different techniques can be taken from (Marx et al., 1996; Möllney et al., 1999). Today a carbon labeling experiment can be performed with a relatively low amount of time, money and manpower. 
Like all other model-based tools the assumptions of a carbon flux model must be carefully inspected. First the assumption of stationary fluxes must now be related to each single cell within the population and not to the population average. Small fluctuations can be tolerated while large fluctuations will lead to misinterpretations of the measured data. The reason is that there is a nonlinear relation between fluxes and measured data which does not behave well with respect to averaging operations. However, up to now the method has always been applied in a continuous culture so that the physiological variety within the population is rather small.

Another assumption is that enzymes cannot distinguish between differently labeled species of a certain substrate. In other words, labeled molecules are converted at the same rate as unlabeled molecules. In contrast, it is well known that isotope mass effects really occur (O'Leary, 1982). However, all these examples deal with $\mathrm{C}_{1}$-bodies mostly in the gas phase. Thus as soon as there are metabolites with more than one carbon atom in the liquid phase the mass effects are unmeasurably small.

Based on these two assumptions the carbon flux balance equations can be formulated. To this end, the system labeling state is described by isotopomer fractions. An isotopomer of a metabolite with $n$ carbon atoms is one of the $2^{n}$ different labeling states in which the metabolite can be encountered (Figure 5). The corresponding isotopomer fractions denote percentages relative to all molecules of this metabolite and thus they sum up to one. Now all isotopomer fractions of all intracellular metabolites are composed to the labeling state vector $\mathbf{x}$, which has a quite high dimension of more than 1000 for a realistic network. Likewise $\mathbf{x}^{\text {inp }}$ is the known vector of all isotopomer fractions in the input substrates fed into the system. The relation between the fluxes and the emerging isotopomer fractions is given by the isotopomer labeling balances

$$
f\left(\mathbf{v}, \mathbf{x}^{\text {inp }} ; \mathbf{x}\right)=\mathbf{0}
$$

which are described in detail in (Wiechert et al., 1999).

Finally, it must be noted that ${ }^{13} \mathrm{C}$ metabolic flux analysis is sensitive to the forward and backward fluxes of bidirectional reaction steps (Wiechert and de Graaf, 1997) (Figure 5). This has the advantage that more information about the metabolic system can be obtained than with stoichiometric flux analysis which solely relies on the net fluxes. In particular, the transaldolase and transketolase steps in the pentose phosphate pathway expose large exchange fluxes which can be 
quantified to some extent (Wiechert et al., 1997). On the other hand if the bidirectionality of these steps is neglected the flux model becomes incorrect and a large flux estimation error can occur (Wiechert et al., 1997). This in turn forces all potentially bidirectional reaction steps to be modeled with two directions which produces a significant increase of unknown flux parameters. Thus a large amount of measured data is required to balance this missing information.

Isotopomer labeling systems are a rare biological example where a large model emerges from just a few modeling principles and basic laws. The deeper reason is that the laws of label distribution over the network do not really have a biological but rather a physical origin. Because the laws of physics usually have a universal scope and are well validated by many experiments the simulation of isotopomer labeling experiments really has a strong predictive power. On the other hand, the model predicts nothing more than the outcoming labeling distribution for known metabolic fluxes, i.e. the "physical" state of the system is predicted from a known "biological" state.

\section{Developed tools}

1. Automatic equation generation: It is completely impossible to specify the large number of equations in (2) in a purely manually way without producing a lot of typing errors. Moreover, frequent alterations in the network will lead to a reworking of more than 1000 equations. For these reasons powerful software tools are required to generate the equations automatically from the known carbon transitions of the reaction steps (Figure 5). Two different approaches are published which are based on the specification of atom mapping matrices (Zupke and Stephanopoulos, 1994) and on a textual notation of the carbon atom transitions (Marx et al., 1996). From this input all other equations are generated (Schmidt et al., 1997; Wiechert et al., 2001).

2. Simulation of labeling experiments: The only purpose of carbon flux models is metabolic flux analysis. To this end a labeling experiment must first be simulated for given fluxes. The solution of the equation system (2) with respect to the unknown labeling state $\mathbf{x}$ for given flux values $\mathbf{V}$ and input labeling $\mathbf{x}^{\text {inp }}$ poses a formidable computational problem. Iterative solution algorithms for this equation system (Schmidt et al., 1997) always suffer from con- 
vergence problems in case of large exchange fluxes. A general analytic solution to the problem was found quite recently (Wiechert et al., 1999). Based on this result an efficient solution algorithm could be implemented that now solves the equation system in about 1 second. Moreover, the sensitivity matrix $d \mathbf{x} / d \mathbf{v}$ is computed in just another second. This makes the simulation of carbon labeling experiments for known fluxes a computationally cheap task.

3. Flux estimation: Flux estimation from given measurements is done by parameter fitting based on successive simulation steps. The fluxes are varied by an iterative solution algorithm until the measured data are best reproduced by the simulation in the sense of least squares (Schmidt et al., 1999; Möllney et al., 1999; Wiechert, 2001). Alternative approaches are based on explicit solutions of the equation system (2) for specific network topologies and experimental conditions (Sauer et al., 1997; Klapa et al., 1999; Park et al., 1999). The computed sensitivities help to implement powerful rapidly convergent optimization algorithms based on gradients.

4. Statistical analysis and experimental design: Recently all statistical tools have been generalized from stoichiometry-based flux analysis to ${ }^{13} \mathrm{C}$ flux analysis (Möllney et al., 1999): the structural identifiability of the fluxes can be decided without knowing the data, all fluxes can be efficiently computed with efficient usage of redundant information, a confidence region for the estimated fluxes can be computed, and gross measurement errors can be detected. Moreover, a powerful experimental design strategy has been developed that helps to find the most "informative" mixture of labeled substrates. By applying this strategy all forward and backward fluxes in the anaplerosis of $C$. glutamicum have recently been quantitated (Petersen

et al., 2000). Summarizing, the ${ }^{13} \mathrm{C}$ technique has nowadays reached the same state of maturity as the stoichiometry-based technique, which is quite an exceptional situation in nonlinear systems theory.

\section{Stationary Mechanistic Models}




\section{Basic assumptions}

In contrast to stoichiometric models, mechanistic approaches to metabolic modeling incorporate the regulatory structures that lead to a certain flux distribution. Thus a valid mechanistic model should not only produce fluxes that obey the stoichiometric relations but also explain in some sense why this intracellular flux pattern emerges. Moreover, if a valid model has some predictive power because it tells us how the intracellular fluxes will change when the external substrate concentrations or some enzymes are altered. Nevertheless, a mechanistic model can only predict the effect of alterations which are explicitly contained in the model.

A basic assumption for mechanistic modeling is that all intracellular metabolites are homogeneously distributed within their respective cellular compartments (Figure 1). Thus the effect of possible intracellular concentration gradients or other "spatial" effects are neglected. The validity of these assumptions is seldom discussed although it has dramatic consequences on the validity of models. Likewise - because mechanistic models are highly nonlinear - they must all be considered as "single-cell models", i.e. there is no population variety.

The conceptual basis for mechanistic modeling is the composition of a metabolic network from single enzyme-catalyzed reaction steps (Figure 2). The function of these steps is then described by formulas taken from enzyme kinetics. If whole pathways are lumped into one formal reaction step its function is described by a phenomenological "formal kinetic" term, which is also motivated by the typical enzyme behavior. As an extreme case the whole cell metabolism is condensed into one formal reaction step which leads to the classical Monod or Pirt models of cellular growth (Figure 6). All levels of detail can be imagined between this oversimplified approach and a description of every single reaction step in the system (Sonnleitner and Käppeli, 1986; Domach et al., 1984; Tomita et al., 1999). A signal based hierarchical modeling concept is described in (Gremling, 2000). The top-down decomposition of complex networks into so-called modules is also an important topic in metabolic control analysis (Brown, 1990; Schuster et al., 1993).

If reaction steps are modeled on the basis of enzyme kinetics another assumption must be discussed which is the validity of in vitro enzyme reaction mechanisms under in vivo conditions. It is generally accepted that the reaction mechanism of an enzyme (i.e. active sites, ligand bindings 
and conformational changes) does not change under in vivo conditions so that the enzyme kinetic terms can be transferred to the living cell. On the other hand, the kinetic constants may vary within orders of magnitude because the intracellular environment differs strongly from standardized in vitro conditions (Richey et al., 1987).

There is still no indisputable evidence for the existence or nonexistence of channeling phenomena in the cell cytosol (Mathews, 1993; Sumegi et al., 1993; Agius and Sherrat, 1996). If nonmembrane-bound enzyme complexes play an important role under in vivo conditions the structure of kinetic terms changes because the intermediate metabolite concentration is raised in the neighborhood of complexes (Cornish-Bowden, 1996). This contradicts the assumption of homogeneously distributed metabolites. The consequences of channeling on metabolic control are discussed in (Kholodenko et al., 1996). A phenomenon which is closely related to channeling is macromolecular crowding which may change reaction kinetics significantly (Garner, 1996; Rohwer et al., 1998).

Because precise kinetic formulas are missing for many enzymes simplified or phenomenological approaches are frequently used to facilitate modeling. A well known approach is the power law formalism which uses an exponential expression for each reaction step (Voit, 1991). Another frequently used approximation is to represent a reaction rate by multiplicative saturation and inhibition terms. Finally, thermodynamic flow-force relationships are a way to relate thermodynamics to kinetics (Westerhoff, van Dam, 1987; Nielsen, 1997).

If phenomenological relations (i.e. formal kinetics) are introduced into a model it must be clear that this strongly reduces the potential predictive power of the model. Phenomenological relations like the Monod law are constructed in a purely empirical way from a given data set (for example from an X-D diagram). If a microorganism grows under different conditions the phenomenological law can quickly become invalid.

In the (quasi-)stationary case the system is studied under the condition of constant reaction rates. Accepting all the assumptions made above this leads to a general model structure which extends the stoichiometric model (1):

$$
\mathbf{N} \cdot \mathbf{v}(\boldsymbol{\alpha}, \mathbf{S}, \mathbf{E} ; \mathbf{X})=\mathbf{0}
$$


Here $\boldsymbol{\alpha}$ denotes the vector of all enzyme kinetic parameters, $\mathbf{S}$ is the vector of extracellular substrate concentrations, $\mathbf{E}$ is the vector of (active) enzyme concentrations and $\mathbf{X}$ denotes the vector of intracellular metabolite concentrations. Given $\boldsymbol{\alpha}, \mathbf{S}, \mathbf{E}$ the equation system can be numerically solved for the intracellular state which yields $\mathbf{X}=\mathbf{X}(\boldsymbol{\alpha}, \mathbf{S}, \mathbf{E})$.

\section{Available data}

A lot of data is required to parametrize a mechanistic model. If complex reaction steps with many effectors like the phosphofructokinase system are involved an enzyme kinetic formula soon has 10 or more kinetic parameters (Hofmann and Kopperschläger, 1982). Moreover, the kinetic law is seldom published for the cooperative action of all known effectors because major substrates, cofactors and effectors are usually studied separately.

The kinetics of some important processes like oxydative phosphorylation is almost completely unknown so that modeling assumptions about these metabolic processes are highly speculative. The situation is even worse with transport steps over the various cellular membranes (Krämer, 1996). For example detailed quantitative in vivo data about the various intermediate steps involved in phosphotransferase system is only recently becoming available (Rohwer, 2000). In general the spectrum of all effectors in one enzymatic step is only incompletely known. In silico docking analysis (Westhead et al., 1997) may help to find all the potential ligands of an enzyme in the future.

Last but not least the published data usually stems from different microorganisms or different strains and it was produced over several decades. Thus it is currently almost impossible to obtain a consistent data set for a specific strain of some microorganism. Consequently, it is not surprising that published kinetic parameters for one enzyme differ by orders of magnitudes. For example a variety of $\mathrm{K}_{\mathrm{m}}$-values for the substrate of phosphofructokinase is published in (Ewings and Doelle, 1980) depending on environmental conditions that cannot even measured in vivo.

There is one set of parameters which can never be taken from published data. The enzyme activities $\mathbf{E}$ corresponding to the $v_{\max }$ values of all reaction steps in the network depend on the 
physiological state of the system and the corresponding enzyme expression level. Little is known about the fraction of irreversibly inactivated enzymes and the enzyme degradation process (Rivett, 1986). Although DNA chips offer the opportunity to measure the concentrations of specific mRNA species in vivo it is difficult to relate this data to the gene transcription and translation rate which are also influenced by RNA stability and posttranslational control. Consequently, the vector $\mathbf{E}$ must still be estimated from experimental data like cell extracts and gel electrophoresis. Both techniques suffer from strong disadvantages (enzyme inactivation by cell disruption, unknown purification factors) so that the $\mathbf{E}$ values estimated by this procedure are only precise within an order of magnitude.

\section{Developed tools}

Once a valid mechanistic model of cellular metabolism is available - which is not to be expected in the near future for the above reasons - a "virtual" cell will be available. However, the scope of such a metabolic model is still restricted to physiological states with the same gene expression pattern because $\mathbf{E}$ is considered as a constant. Nevertheless, if the goals are less ambitious the analysis of metabolic models already produces a lot of insights into cellular regulation. These qualitative results are frequently quite insensitive with respect to the parameter values:

1. Modeling: The development of large models must be supported by tools that help to keep track of all the structural assumptions and enzyme kinetic terms in the model. Large metabolic models consist of 50 and more equations and thus are practically unmanageable. Consequently, various computer aided tools for metabolic modeling have been developed which are compared in (Heinrich and Schuster, 1996). They are based on biochemical reaction networks, metabolic pathways, system theoretical concepts (Kremling et al., 2000) or even a rigorous whole cell approach (Tomita et al., 1999). On the other hand current all purpose simulation frameworks like MODELICA or gPROMS become more and more powerful.

2. Simulation: Based on the fully specified model different physiological scenarios can be simulated. Although the available algorithms for the solution of general nonlinear equation systems are quite powerful today this is still a computationally expensive and difficult prob- 
lem. In particular the case of multiple solutions may arise where different flux patterns occur for the same external conditions $\mathbf{S}$. It is still an empirical procedure to find all these states.

3. Metabolic control analysis: Once a solution to the equation system (3) has been computed, the influence of parameter variations (i.e. of $\boldsymbol{\alpha}, \mathbf{S}, \mathbf{E}$ ) on the system state can be analyzed. This is done by computing sensitivity coefficients $d \mathbf{v} / d \boldsymbol{\alpha}, d \mathbf{v} / d \mathbf{S}, d \mathbf{v} / d \mathbf{E}$ and is facilitated by modern computer algebra systems which can automatically derive the model equations with respect to all the parameters (Griewank, 2000).

Sensitivity coefficients can be better interpreted if they are expressed on a percentage scale as relative sensitivities. This leads to the control coefficients as defined by metabolic control theory (Fell, 1997). Metabolic control analysis has become the most widely used tool to gain a quantitative understanding of metabolic networks. Moreover there is a well developed theory behind MCA (Reder, 1988; Heinrich, Schuster, 1996) accompanied by a well established terminology which facilitates to communicate about metabolic control (Kell, Westerhoff, 1986). The most important result of metabolic control theory for metabolic engineering is that the control of a complex metabolic network is usually distributed over many different enzymatic steps. This has the consequence that there is little chance for a single genetic modification to result in a large chance of the flux distribution (Niederberger et al, 1986).

From the computed control coefficients it can be immediately derived which parameters strongly influence the current flux pattern and which parameters play a minor role and thus need not be known precisely. However, a reaction step which is unimportant in one physiological state may well be important in another state (Pissara et al., 1996). Model-based MCA has been applied to many different reaction networks from simple case studies (Hofmeyr, 1989) to whole cellular subsystems like glycolyis (Cascante et al., 1995) or the citric acid cycle (Torres, 1994b).

The experimental in vivo determination of control coefficients can be a helpful tool for modeling and model validation. A number of perturbation methods have been developed for this task (Fell, 1997). The application of experimental MCA methods to Metabolic Engineering is 
described in (Kacser, Acerenza, 1993). However it is still difficult to measure all the required metabolite concentrations in vivo. As a more pragmatic approach different pool lumping concepts have been developed like top-down MCA (Brown et al., 1990), hierarchical control analysis (Kahn, Westerhoff, 1991) or group control coefficients (Simpson et al., 1998).

4. Branch node classification: A method that is closely related to sensitivity analysis is the concept of rigid and flexible branch points in a metabolic network (Stephanopoulos, Vallino, 1991). A branch point is said to be rigid if the split ratio of the branch fluxes is tightly controlled, otherwise it is called flexible. For example a node becomes rigid if each branch is crossregulated by the other. The classification of branch points by qualitative network analysis, kinetic models and flux analysis (Vallino, Stephanopoulos, 1994) is a promising tools for metabolic engineering.

5. Large parameter variations: Sensitivity coefficients are in general not suitable for predicting the change of the fluxes with respect to large variations in the parameters, as for example the overexpression of an enzyme. In order to investigate the behavior for large changes parameter variations over orders of magnitudes have to be performed (Schuster, Holzhütter, 1995). From a computational perspective so-called continuation methods are well suited to solve this problem (Allgower and Georg, 1990). These methods track the solution while the parameters are varied continuously. In this tracking process bifurcations can occur or the solution can even cease to exist. Such phenomena give valuable insights into the system behavior because they restrict the possible range of physically meaningful parameters. Consequently, this tool is valuable for qualitative model validation.

Another approach is the extension of sensitivity coefficients for large parameter variations. A second order Taylor series expansion is described in (Höfer, Heinrich, 1993). Simplifications of the kinetic rate functions are suggested in (Small, Kacser, 1993a+b).

6. Optimal regulatory architectures: The most ambitious application of metabolic models is the computation of optimal parameters for $\boldsymbol{\alpha}, \mathbf{S}, \mathbf{E}$ as long as the gene expression pattern remains unchanged (Torres et al., 1996; Hatzimanikatis et al., 1996). In practice these parameters can be altered by overexpressing or knocking out genes, by genetically altering a protein 
structure or by changing the external conditions. Typically the optimization criterion is the maximal product formation or maximum product yield. The optimum is computed by optimization algorithms which can operate on a restricted search space with mixed continuous and integer parameters (in general genes can only be overexpressed by integer factors). However, such results are currently highly speculative.

\section{Nonstationary Mechanistic Models}

\section{Basic assumptions}

If the short-time behavior of microorganisms has to be described under rapidly changing external conditions nonstationary models are required. Such rapid transients can be the effect of a substrate pulse experiment or the inhomogeneous conditions within a large-scale bioreactor. In both cases time constants in the order of 0.1-10 seconds are at the focus of interest. The general nonstationary metabolic model for this situation extends the stationary model (3) by differential terms:

$$
\dot{\mathbf{X}}=\mathbf{N} \cdot \mathbf{v}(\boldsymbol{\alpha}, \mathbf{S}, \mathbf{E} ; \mathbf{X})
$$

The pool sizes and reaction rates are no longer assumed to be constant and their dynamic behavior is described explicitly. In particular the components of $\mathbf{v}$ are now called reaction rates rather than fluxes. The stoichiometric relations (1) do not hold in the nonstationary situation. Thus (4) is the most general model because it contains the previous models as special cases.

Once more the required modeling assumptions must be critically discussed. Basically they are the same as for stationary modeling. However, on the short-time scale more intracellular processes

may have an effect because all metabolite pools are simultaneously excited. Fortunately, gene regulatory processes are irrelevant on this time scale.

\section{Measured data and developed tools}


Currently, the predominant application of nonstationary models is the evaluation of rapid sampling experiments (van Dam and de Koning, 1992; Theobald et al., 1997). This technique has made considerable progress in recent years and nowadays the concentrations of about 15 intracellular metabolites can be monitored at a sampling rate of $5 \mathrm{~Hz}$ over a time span of $35 \mathrm{sec}$ (Schaefer et al., 1999) (Figure 7). This gives a total of more than 2500 samples which require an automated process for sampling, rapid cell inactivation by methanol quenching, cell disruption and chemical analysis. This is achieved by extensive automation and the use of a laboratory robot. The quality of the measured data is quite high as was shown by repeating an experiment under the same conditions.

1. Simulation: Clearly, the available computer aided modeling tools for the stationary case can also used for the nonstationary case. Given the model and assumed parameter values the nonstationary cellular response can be computed by a differential equation or algebraic differential equation solver. This is not always an easy task because the number of equations is quite large and stiffness problems caused by different time constants frequently occur in metabolic systems (Ascher and Petzold, 1998).

2. Data evaluation: Given a data set and a nonstationary model this setting gives the unique opportunity of estimating most parameters in the model from measured in vivo data (Rizzi et al., 1997). In particular the in vivo enzyme activity constants $\mathbf{E}$ can be estimated. However, this is an extremely complex parameter fitting problem which frequently suffers from missing measurement information, slow or poor convergence of the optimization algorithms, and multiple local optima. Currently this cannot be managed without taking some of the parameters from the literature. A new evaluation strategy is described in (Visser et al., 2000).

3. Systems analysis: A unique feature of dynamic models is their ability to describe the global dynamic behavior of a system. In particular the occurrence of stable stationary states, multiple stationary states and oscillations are of great interest for the understanding of a dynamic system (Jordan, Smith, 1987). In the biological application dynamic system analysis is a tool for qualitative model validation because the model must at least exhibit the same dynamic behavior as the real system. Typical examples are stability analysis (Torres, 1994a) or the 
analysis of oscillations (Wolf et al., 2000). In combination with bifurcation analysis the influence of parameters on the global system behavior can be studied (Wolf et al., 2000).

\section{Models with Gene Regulation}

\section{Basic assumptions and missing data}

None of the models discussed so far does account for genetic regulation (Figure 8). The gene regulation network is responsible for the gene expression rate and thus for the establishment of certain enzyme activities in the metabolic network. As a major difference between the metabolic and genetic regulatory networks the time constants of gene regulatory mechanisms (about 1 hour) are much larger than the time constant of metabolic regulatory mechanisms (about 10 seconds). The structural and quantitative understanding of the overlaid genetic regulation network is currently only in its infancy.

In principle, the genetic apparatus of a cell is - like metabolism - based on ligand-binding mechanisms and chemical reaction steps (Figure 9). For this reason it may be represented by some generalized reaction network. However, the number of elementary binding and reaction steps that take part in a single protein's translation, transcription, activation, repression, inactivation and degradation is so great that a simplified approach must be chosen. Typically, translation, transcription and inactivation are modeled by simple formal kinetics while the gene regulatory mechanisms are represented with more detail (Lee and Bailey, 1984a,b).

A special problem for the description of gene regulation are the inherent stochastic phenomena in the mechanisms of gene activation and repression. Regulatory proteins can be present with only a few copies per cell and likewise the protein effectors can have very low concentrations. Consequently, the approximation of a protein pool by a concentration value is no more valid. When gene regulatory mechanisms are excited as for example in the diauxic shift of $S$. cerevisiae (Derisi et al., 1997) this may lead to a nonhomogeneous population. In one part of the cells certain 
structural genes may already be switched on while they are still switched off the other cells. Once more this makes the averaged measured data hard to interpret.

A major obstruction to the development of quantitative models for gene regulation is the lack of knowledge even at the structural level. In particular, there are regulatory hierarchies within gene regulation like regulons and operons. Hopefully, the interactions of structural and regulatory genes will be resolved in the future by functional genomic methods. It is wise to start with procaryotic gene regulation because this is the least complex case. However, even if the gene regulatory structure is known we are far from quantitative knowledge. Quantitative data about expression rates, binding affinities or enzyme inactivation and degradation are at best orders of magnitudes (Lee and Bailey, 1984a,b).

\section{Modeling approaches}

For the given reasons all currently published modeling approaches are highly speculative and a general model structure like $(3,4)$ for metabolic networks is not in sight. Thus only some basic ideas shall be discussed here:

1. Phenomenological approach: Pure phenomenological models of gene expression take the enzyme activities in the different observed physiological states as constants that have to be determined from experimental data. There is no explanation of how the enzyme activities are regulated. The shift from one physiological state to another is described by a simple first order set point controller. This introduces another time constant, i.e. the proportionality factor of the controller. As an example the diauxic shift of $S$. cerevisia from glucose to ethanol usage has been described in this way (Sweere et al., 1988).

2. Mechanistic approach: A more detailed model of a single enzyme's expression process represents the gene regulatory mechanism in detail but summarizes gene translation, transcription, inactivation and degradation by some few phenomenological terms. The different ligand binding steps are modeled by standard mass action kinetics (Figure 9). Even for one regulated structural gene with an activator and a repressor protein more about 15 different binding con- 
stants are required (Agger and Nielsen, 1999). Certain proteins like RNA polymerase expose a particularly complex gene expression pattern which lead to even more complex models (Axe and Bailey, 1994). More than 100 genes are modeled in the E-CELL project (Tomita et al., 1999). The hierarchical signal-based modeling approach of (Kremling, Gilles, 2001) also incorporates gene regulation. The complexity of all these models shows that the attempt to incorporate gene expression on a single gene basis leads to another very large parameter set. Thus it is important to disentangle the regulatory hierarchy so that gene regulatory models can be restricted to groups of commonly regulated enzymes.

3. Metabolic optimization criteria: The concept of metabolic optimization criteria is based on the assumption that in the process of evolution certain criteria have been maximized. The philosophical question about the correctness of this concept will not be entered here. However, if the optimization concept is accepted then only some few criteria, as for example maximal growth rate or growth yield, maximal flux, maximal substrate uptake, minimal intermediate concentrations, substrate uptake or ATP production, minimal transient times or maximal thermodynamic efficiency, make sense. They are compared in (Heinrich and Schuster, 1996). Although this list might not be exhaustive it is reasonable to assume that evolution has reached one of these goals or a combination of goals. This leads to an augmentation of the metabolic model (2) by a metabolic optimization criterion

$$
\operatorname{MOC}(\boldsymbol{\alpha}, \mathbf{E}, \mathbf{X})=\max
$$

Evaluating this criterion will lead to a deterministic dependence between the enzyme activities, the kinetic parameters and the metabolite concentrations. Clearly, - in order to obtain a well defined solution - the energy effort to synthesize proteins (which is more than $50 \%$ in growing cells) must be incorporated in the metabolic network and the substrate uptake must be limited. If these efforts are taken into account there will be a limited energy budget for all intracellular processes so that the cellular regulation must find a proper balance between catabolism and anabolism.

A technical advantage of metabolic optimization criteria is that an evolutionary construction of metabolic models is greatly simplified. The emphasis can be first laid on a certain part of the network where detailed enzyme kinetic terms are used. The rest is roughly described by 
formal kinetics or some metabolic fluxes may even be left unspecified. The optimization operation will then find a - hopefully reasonable - solution which makes it possible to simulate a network with little knowledge. This is quite an important feature because modeling is usually an iterative process in which simulation scenarios produce the right ideas for the next model improvement.

4. Cybernetic modeling: A more systematic optimization approach is that of cybernetic modeling where the metabolic optimization criterion is defined in a purely formal procedure (Varner and Ramkrishna, 1999). The cybernetic approach is based on the idea of pathway evolution that has its motivation in commonly regulated enzyme groups (operons). It is postulated that pathway evolution always has the goal of optimizing the formation of the pathway product (e.g. pyruvate in glycolysis). On the other hand, different pathways compete for intracellular resources. This leads to a hierarchically defined optimization criterion with an overall goal (growth) and several subgoals (pathway resources). The only decision that is left to the modeler is the specification of pathways. From a computational viewpoint cybernetic modeling poses some difficult problems because a hierarchically defined nonsmooth criterion has to be maximized.

Clearly, the application of models with gene regulation is currently restricted to exploratory simulation, the understanding of basic gene regulatory mechanisms or rough predictions of the order of magnitude. General modeling tools are currently not available. Moreover, the optimization problems arising can be quite complicated because they contain nondifferentiable terms due to the minimization operation.

\section{Central Problems and Future Developments}

At the beginning of this review a comparison between metabolic networks and electrical circuits was made. It has become clear that there are principal obstructions for a direct transfer of established methods from the classical engineering sciences to metabolic engineering (population variety, in vitro-in vivo transfer of kinetic parameters, diversity of mechanisms, missing structural 
knowledge). Consequently, the computer aided design of metabolic networks is an ambitious goal if not illusionary at all. However, models and software tools are already an essential part in metabolic engineering. They are extremely helpful to organize the available metabolic knowledge and to design the right experiments. Nevertheless the decisions about genetic manipulations must still be made by the human experts. As a conclusion some crucial problems of tool development based on metabolic models are summarized that may serve as a guideline for future developments:

\section{Consistent database}

Although many database systems for metabolome, proteome and transcriptome data are currently under construction (Hofestädt, 2000), model building is far from being a simple "cut and paste" procedure. Even if software interfaces were available which automatically translate database information into models the result would be extremely poor. The reason is the enormous heterogeneity of this database. Over several decades different types of experiments have been performed for different organisms and strains by different research groups and with different measurement and evaluation methods. Currently there is not even a consistent and complete data set for the central metabolic pathways of for E. coli K12.

The problem becomes even worse when large amounts of in vivo data produced by flux analysis, rapid sampling, gel electrophoresis and DNA chips are added to this data set. These in vivo data sets are not standardized and thus not archived in databases. This will almost surely produce further inconsistencies which will lead to severe problems in the model-based interpretation of the data.

\section{Genetic regulation}

The quantitative and even structural description of gene regulatory mechanisms is still in its infancy. A lot of quantitative data about gene regulation, translation and transcription on the one and protein inactivation and degradation on the other hand is required to formulate appropriate 
models. Additionally, jointly regulated genes must be identified to obtain the necessary information for model simplification.

\section{Validation of large models}

Model validation has been identified as the most critical part in the modeling process and some statistical methods have been mentioned. The difficult problem now is the extension of these methods to large metabolic models with many alternatives and numerous kinetic constants. Indeed the rigorous extension of the available mathematical algorithms for small models lead to enormously complex optimization problems which are far beyond current computational power even on supercomputers. Thus heuristic algorithms for large model validation and simplification must be developed which do not find the globally best solution to the discrimination problem but a practically acceptable one.

One building block of the model validation process is the fitting of complex nonlinear models to large data sets. Although many algorithms have been published for this task an automatic parameter fitting procedure is still a long way off. Instead a good initial guess and a lot of manual intervention is required to evaluate large data sets. The reason for these problems is that all algorithms work well only in the case where the model really can describe the data and there are no outliers or even inconsistent parts in the data set. Otherwise considerable convergence problems or unsatisfactory solutions will occur. Thus more robust algorithms are required to evaluate the data. It would even be a great success to prove that a certain model cannot explain the data.

\section{Multidisciplinary research}

The solution of analytical or computational problems and the development of software systems plays a central part in the process of tool development for metabolic engineering. It has become clear that in contrast to classical bioengineering models we are now dealing with a considerably larger amount of equations. These can definitely not be managed with classical ad hoc methods because efficient algorithms for model generation, analysis and equation solution are now re- 
quired for problem solution. This requires extensive skills which are usually not available in the biotechnology or bioengineering community. Thus multidisciplinary cooperation is required for successful tool development. Many disciplines, among them scientific computing, statistics, discrete mathematics, nonlinear systems theory, bioinformatics, simulation and software engineering, can contribute to this development.

\section{Investigation of single cells}

A lot of modeling assumptions have been mentioned like the quasi-stationarity of single cells or populations, homogeneously distributed metabolites, nonexistence of stochastic phenomena and so on. These assumptions are commonly made in the metabolic modeling community and are seldom discussed critically. Clearly, if one of these crucial assumptions were strongly invalid then the prediction of metabolic behavior would be an even more difficult problem.

In a critical scientific attitude efforts should be undertaken to achieve some insight into single cells and to characterize the population variety more precisely. Only a few methods like flow cytometry are currently available to get some information. For large mammalian cells microtubes can be used to inject substances directly into the cell. As a matter of fact intracellular diffusion i.e. spatial inhomogenity - plays an important role in these cells (Schaff and Loew, 1999). Possibly micro- and nanomechanical methods can help to achieve some insight into smaller cells in the future (Buer et al., 1998).

Another approach is the exploratory attempt to build single cell simulators which take the spatial structure into account. This can be done on the basis of geometrical models (Dawson et al., 2000), models distributed over space (Schaff and Loew, 1999) or particle models. Without doubt such simulations will require supercomputing power. They might produce some indications of possible intracellular phenomena that cannot be observed from average cell population data. 


\section{References}

Agger, T., Nielsen, J., 1999. Genetically structured modeling of protein production in filamentous fungi. Biotechnol. Bioeng. 66(3), 164-170.

Agius, L., Sherrat, H. (Eds.), 1996. Channeling in intermediary Metabolism. Portland Press.

Albe, K., Wright, B., 1992. Systems analysis of the tricarboxylic acid cycle in Dictyostelium discoideum: II. Control analysis. J. Biol. Chem. 267, 3106-3114.

Allgower, E.L., Georg, K., 1990. Numerical Continuation Methods, Springer.

Ascher, U., Petzold, L., 1998. Computer Methods for Ordinary Differential Equations and Differential-Algebraic Equations. SIAM.

Axe, D., Bailey, J., 1994. Modeling the regulation of bacterial genes producing proteins that strongly influence growth. Biotechnol. Bioeng. 43, 242-257.

Bailey, J.E., 1991. Towards a science of metabolic engineering. Science 252, 1668-1674.

Bonarius, H.P.J., Schmidt, G., Tramper, J., 1997. Flux analysis of underdetermined metabolic systems: The quest for missing constraints. Trends Biotechnol. 15, 308-314.

Box, G., Hill, W., 1967. Discrimination among mechanistic models. Technometrics 9(1), 57-71.

Brand, M.D., 1994. The stoichiometry of proton pumping and ATP synthesis in mitochondria. The Biochemist 16, 20-24.

Brown, G.C., Hafner, R.P., Brand, M.D., 1990. A 'top-down' approach to the determination of control coefficients in metabolic control theory. Eur. J. Biochem. 188, 321-325. 
Buer, S., Gahagan, K.T, Swartzlander, G.A., Weathers, P.J., 1998. Insertion of microscopic objects through plant cell walls using laser microsurgery. Biotechn. Bioeng., 60: 348-355.

Cascante, M., Curto, R., Sorribas, A., 1995. Comparative Characterization of the Fermentation Pathway of Saccharomyces cerevisiae using Biochemical Systems Theory and Metabolic Control Analysis: Steady-State Analysis. Mathematical Biosciences. 130, 51-69.

Cellier, F., 1991. Continuous System Modeling. Springer.

Clarke, B., 1988. Stoichiometric network analysis. Cell Biophys. 12, 237-253.

Collado-Vides, J., 1989. A transformational-grammar approach to the study of the regulation of gene expression. J. Theor. Biol. 136.

Cornish-Bowden, A., Wharton, C., 1988. Principles of Enzyme Kinetics. Oxford University Press.

Cornish-Bowden, A., 1996. Kinetic consequences of channeling. In: Agius, L., Sherrat, H.S.A., (Eds.), Channeling in intermediary Metabolism, pp. 53-70, Portland Press.

Dawson, K., Devlin, T., Klymkowsky, M., Rochev, Y., Snyder, M., Steer, M., Widom, J. (Eds.), 2000. The Dynamic Cell. Springer.

Derisi, J., Iyer, V., Brown, P., 1997. Exploring the metabolic and genetic control of gene expression on a genomic scale. Science 278, 680-686.

Domach, M., Leung, S., Cahn, R., Cocks, C., Shuler, M., 1984. Computer Model for GlucoseLimited Growth of a Single Cell of Eschericia coli B/r-A. Biotechnol. Bioeng. 26, 203-216.

Edwards, J., Palsson, B., 1998. How will bioinformatics influence metabolic engineering? Biotechnol. Bioeng. 58, 162-169. 
Eschenauer, H.A., Koski, J., Osysczka, A., 1990. Multicriteria Design Optimization - Procedures and Applications. Springer.

Ewings K.N., Doelle, H.W., 1980. Further kinetic characterization of the non-allosteric phosphofructokinase from Escherichia coli K-12. Biochem. Biophys. Acta 615(1), 103-112.

Fell, D., 1997. Understanding the Control of Metabolism. Portland Press.

Fitton, V., Rigoulet, M., Ouhabi, R., Guérin, B., 1994. Mechanistic stoichiometry of yeast mitochondrial oxidative phosphorylation. Biochemistry, 33, 9692-9698.

Garner, M. M., 1996. The consequences of macromolecular crowding for metabolic channeling. In: Agius, L., Sherrat, H.S.A. (Eds.), Channeling in intermediary Metabolism, pp. 41-52. Portland Press.

Goldbeter, A., 1996. Biochemical oscillations and cellular rhythms, Cambridge University Press.

Griewank, A., 2000. Principles and Techniques of Algorithmic Differentiation. SIAM.

Hatzimanikatis, V., Floudas, C., Bailey, J., 1996. Optimization of regulatory architectures in metabolic reaction networks. Biotechnol. Bioeng. 52, 485-500.

Hatzimanikatis, V., Choe, L.H., Lee, K.H., 1999. Proteomics: Theoretical and experimental considerations. Biotechnol. Prog. 15, 312-318.

Heinrich, R,, Schuster, S., 1996. The Regulation of Cellular Systems. Chapman \& Hall.

Höfer, T., Heinrich, R., 1993. A second-order approach to metabolic control analysis. J. Theor. Biol. 164, 85-102.

Hofestädt, R., 1993. A simulation shell to model metabolic pathways. Journal of Systems Analysis Modelling Simulation 11, 253-262. 
Hofestädt, R. (Ed.), 2000. Bioinformatik - Forschungsführer Informatik in den Biowissenschaften. Biocom AG.

Hofmeyr, J.-H.S., 1989. Control-pattern analysis of metabolic pathways. Flux and concentration control in linear pathways. Eur. J. Biochem. 200, 223-236.

Hofmeyr, J.-H.S., Olivier, B.G., Rohwer, J.M., 2000. Putting the cart before the horse: Designing a metabolic system in order to understand it. In: Cornish-Bowden, A., Cardenas, M. L. (Eds), Technological and Medical Implications of Metabolic Control Analysis, pp. 299-308. Kluwer Academic.

Hofmann, E., Kopperschläger, G., 1982. Phosphofructokinase in yeast. In: Wood, W.A. (Ed.), Methods in Enzymology. Academic Press, pp. 49-60.

Hohenbalken, B.V., Clarke, B., Lewis, J., 1987. Least distance methods for the frame of homogeneous equation systems. J. Comput. Appl. Math. 19, 231-241.

Jordan, D.W., Smith, P., 1987. Nonlinear Ordinary Differential Equations, Oxford University Press.

Kacser, H., Acerenza, L., 1993. A universal Method for achieving Increases in metabolite production. Eur. J. Biochem. 216, 361-367.

Kahn, D., Westerhoff, H.V., 1991. Control theory of regulatory cascades. J. Theor. Biol. 153, 255-285.

Kanehisha, M., 1999. Post-genomic Informatics. Oxford University Press.

Kao, C.M., 1999. Functional genomic technologies: Creating new paradigms for fundamental and applied biology. Biotechnol. Prog. 15, 304-311. 
Karp, P., Paley, S., 1994. Automated drawing of metabolic pathways. In: Lim, H., Cantor, C., Robbins, R. (Eds.), Third International Conference on Bioinformatics and Genome Research.

Kell, D.B., Westerhoff, H.V., 1986. Metabolic Control Theory: its Role in Microbiology and Biotechnology, FEMS Microbiology Reviews. 39, 305-320.

Kholodenko, B.N., Cascante, M., Westrerhoff, H.V., 1996. Control and regulation of channeled versus ideal pathways. In: Agius, L., Sherrat, H.S.A. (Eds.), Channeling in intermediary Metabolism, pp. 91-114. Portland Press.

Klapa, M.I., Park, S.M., Sinskey, A.J., Stephanopoulos, G., 1999. Metabolite and isotopomer balancing in the analysis of metabolic cycles: I. Theory. Biotechnol. Bioeng. 62, 375-391.

Kohn, M., Lemieux, D., 1991. Identification of regulatory properties of metabolic networks by graph theoretical modeling. J. Theor. Biol. 150, 3-25.

Krämer, R., 1996. Analysis and Modeling of Substrate Uptake and Product Release by Prokaryotic and Eukaryotic Cells. Adv. Biochem. Eng. Biotechnol. 54, 31-74.

Kremling, A., Jahreis, K., Lengeler, J.W., Gilles, E.D., 2000. The organization of metabolic reaction networks: A signal-oriented approach to cellular models. Metabolic Engineering. 2, 190200.

Kremling, A., Gilles, E. D., 2001. The organization of metabolic reaction networks: II. Signalprocessing in hierarchical structured functional units. Metabolic Engineering. 3, 138-150.

Lee, S., Bailey, J., 1984a. Genetically structured models for lac promoter-operator function in the Escherichia coli chromosome and in multicopy plasmids: lac operator function. Biotechnol. Bioeng. $26,1372-1382$. 
Lee, S., Bailey, J., 1984b. Genetically structured models for lac promoter-operator function in the Escherichia coli chromosome and in multicopy plasmids: lac promoter function. Biotechnol. Bioeng. $26,1383-1389$.

Linhart, H., Zucchini, W., 1986. Model Selection. Wiley.

Marx, A., de Graaf, A., Wiechert, W., Eggeling, L., Sahm, H., 1996. Determination of the fluxes in central metabolism of Corynebacterium glutamicum by NMR spectroscopy combined with metabolite balancing. Biotechnol. Bioeng. 49, 111-129.

Mathews, C., 1993. The cell: Bag of enzymes or network of channels? J. Bacteriol. 75(20), 63776381.

Mavrovouniotis, M., 1993. Identification of qualitatively feasible metabolic pathways. In: Hunter, L. (Ed.), Artificial Intelligence and Molecular Biology. AAAI Press.

Mavrovouniotis, M., Stephanopoulos, G., Stephanopulos, G., 1992. Computer-aided synthesis of biochemical pathways. Biotechnol. Bioeng. 36, 1119-1132.

Melendez-Hevia, E., 1994. Optimization of metabolism: the evolution of metabolic pathways toward simplicity through the game of the pentose phosphate cycle. J. Theor. Biol. 166, 201-220.

Michal, G., 1999. Biochemical Pathways - An Atlas of Biochemistry and Molecular Biology. Wiley.

Möllney, M., Wiechert W., Kownatzki, D., de Graaf, A.A., 1999. Bidirectional reaction steps in metabolic networks. Part IV: Optimal Design of Isotopomer Labeling Experiments, Biotechnol. Bioeng., 66(2):86-103.

Neidhardt, F., Ingraham, J., Schaechter, M., 1990. Physiology of the Bacterial Cell - A Molecular Approach. Sinauer Associates. 
Niederberger, P., Prasad, R., Miozzari, G., Kaczer, H., 1986. A strategy for increasing an in vivo flux by genetic manipulations: The tryptophan system of yeast. Biochem. J. 287, 473-479.

Nielsen, J., 1997. Metabolic control analysis of biochemical pathways based on a thermokinetic description of reaction rates. Biochem. J. 321, 133-138.

Nielsen, J., 1998. Metabolic Engineering: Techniques for Analysis of Targets for Genetic Manipulations, Biotechn. Bioeng. 58, 125-132.

O'Leary, M.H., 1982. Heavy-atom isotope effects on enzyme-catalyzed reactions. In: Schmidt, H.-L., Förstel, H., Heinzinger, K. (Eds.), Stable Isotopes, Proceedings of the 4th International Conference, Jülich, March 23-26, 1981. Elsevier, pp. 67-75.

Park, S.M., Klapa, M.I., Sinskey, A.J., Stephanopoulos, G., 1999. Metabolite and isotopomer balancing in the analysis of metabolic cycles: II. Applications. Biotechnol. Bioeng. 62, 392-401.

Petersen S., de Graaf A.A., Eggeling L., Möllney M., Wiechert W., Sahm H. (2000) In vivo quantification of parallel and bidirectional fluxes in the anaplerosis of Corynebacterium glutamicum. submitted.

Pfeiffer, T., Sanchez-Valdenebro, I., Nuno, J., Montero, F., Schuster, S., 1999. METATOOL: For studying metabolic networks. Bioinformatics 15, 251-257.

Pissara, P., Nielsen, J., Bazin, M.J., 1996. Pathway kinetics and metabolic control analysis of a high-yielding strain of Penicillium chrysogeneum during fed batch cultivations. Biotechnol. Bioeng., 51: 168-176.

Popper, K., 1971. Conjectural knowledge: My solution of the problem of induction. Rev. Int. Phil. 25(95-6). 
Reddy, V., Mavrovouniotis, M., Liebman, M., 1993. Petri net representations in metabolic pathways. In: Hunter, L., Searls, D., Shavlik, J. (Eds.), ISMB-93, Proceedings of the First International Conference on Intelligent Systems for Molecular Biology. AAAI Press, pp. 328-336.

Reder, C., 1988. Metabolic Control Theory: A Structural Approach. J.Theor.Biol. 135, 175-201.

ap Rees, T., 1988. Compartmentation of plant metabolism. In: Davies, D.D. (Ed.), The Biochemistry of Plants. Academic Press, pp. 87-115.

Richey, B., Cayley, D.S., Mossing, M.C., Kolka, C., Anderson, C.F., Farrar, T.C., Record Jr., M.T., 1987. Variability of the intracellular ionic environment of Escherichia coli. J. Biol. Chem. $262,7157-7164$.

Rivett, A., 1986. Regulation of intracellular protein turnover: Covalent modification as a mechanism of marking proteins for degradation. Curr. Top. Cell. Regul. 28, 291-337.

Rizzi, M., Baltes, M., Theobald, U., Reuß, M., 1997. In vivo analysis of metabolic dynamics in Saccharomyces cerevisiae: II. Mathematical model. Biotechnol. Bioeng. 55(4), 592-608.

Rohwer, J.M., Schuster, S., Westerhoff, H.V., 1996. How to recognize functional units in a metabolic system, J. Theoret. Biol. 179, 214-228.

Rohwer, J.M., Postma, P.W., Kholodenko, B.N., Westerhoff, H.V., 1998. Implications of macromolecular crowding for signal transduction and metabolite channeling, Proc. Natl. Acad. Sci. US. 95, 10547-10552.

Rohwer, J.M., Meadow, N.D., Roseman, S., Westerhoff, H.V., Postma, P., 2000. Understanding glucose transport by the bacterial phophoenolpyruvate; glucose phosphotransferase system on the basis of kinetic measurements in vitro. J. Biol. Chem. 275, 34909-34921.

Rumbaugh, J., Jacobsen, I., Booch, G., 1997. Unified Modelling Language: Manual. Addison Wesley. 
Sauer, U., Bailey, J.E., 1997. Estimation of P-to-O ratio in Bacillus subtilis and its influence on maximum riboflavin yield. Biotechnol. Bioeng.. 64, 750-754.

Schaefer, U., Boos, W., Takors, R., Weuster-Botz, D., 1999. Automated sampling device for monitoring intracellular metabolite dynamics. Anal. Biochem. 270, 88-96.

Schaff, J., Loew, L., 1999. The virtual cell. In: 4th Pacific Symposium on Biocomputing, pp. 228-239.

Schilling, C.H., Schuster, S., Palsson, B.O., Heinrich, R., 1999. Metabolic Pathway Analysis: Basic Concepts and Scientific Applications in the Post-genomic Era. Biotechnol. Progress. 15, 296-303.

Schmidt, K., Carlsen, M., Nielsen, J., Villadsen, J., 1997. Modelling isotopomer distribution in biochemical networks using isotopomer mapping matrices. Biotechnol. Bioeng. 55(6), 831-840.

Schmidt, K., Nielsen, J., Villadsen, J., 1999. Quantitative analysis of metabolic fluxes in E. coli, using 2 dimensional NMR spectroscopy and complete isotopomer models. Journal of Biotechnology, 71: 175-189.

Schomburg, D. (Ed.), 1997. Enzyme Handbook. Springer.

Schuster, S., Kahn, D., Westerhoff, H.V., 1993. Modular analysis of the control of complex metabolic networks. Biophys. Chem. 48, 1-17.

Schuster, S., Fell, D., Dandekar, T., 1999. A general definition of metabolic pathways useful for systematic organization and analysis of complex metabolic networks. Nature Biotechnol. 18, 326-332.

Seber, G., Wild, C., 1989. Nonlinear Regression. Wiley. 
Seressiotis, A., Bailey, J., 1988. MPS: An artificially intelligent software system for the analysis and synthesis of metabolic pathways. Biotechnol. Bioeng. 31, 587-602.

Shiraishi, F., Savageau, M., 1992. The tricarboxylic acid cycle in Dictystelium discoideum. III. Analysis of steady state and dynamic behaviour. J. Biol. Chem. 267, 22926-22933.

Simpson, T.W., Follstad, B.D., Stephanopoulos, G., 1998. Experimental determination of group flux control coefficients in metabolic networks, Biotechnol. Bioeng. 58, 149-153.

Small, J.R., Kacser, H., 1993. Responses of metabolic systems to large changes in enzyme activities and effectors: I. The linear treatment of unbranched chains. Eur. J. Biochem. 213, 613624.

Small, J.R., Kacser, H., 1993. Responses of metabolic systems to large changes in enzyme activities and effectors: II. The linear treatment of branched pathways and metabolite concentrations. Assessment of the general nonlinear case. Eur. J. Biochem. 213, 625-640.

Sonnleitner, B., Käppeli, O., 1986. Growth of Saccharomyces cerevisiae is controlled by its limited respiratory capacity: Formulation and verificaton of a hypothesis. Biotechnol. Bioeng. 28, 927-937.

Stephanopoulos, G., Vallino, J.J., 1991. Network rigidity and metabolic engineering in metabolite overproduction. Science. 252, 1675-1681.

Stephanopoulos, G., Sinskey, A.J., 1993. Metabolic engineering - methodologies and future prospects. TibTech. 11, 392-396.

Stephanopoulos, G.N., Aristidou, A.A., Nielsen, J., 1998. Metabolic Engineering - Principles and Methodologies. Academic Press.

Stephanopoulos, G., 1999. Metabolic fluxes and metabolic engineering. Metabolic Engineering. 1, 1-10. 
Sumegi, B., Sherry, A.D., Malloy, C.R., Srere, P.A., 1993. Evidence for orientation-conserved transfer in the TCA cycle in Saccharomyces cerevisiae: ${ }^{13} \mathrm{C}$ NMR studies. Biochemistry 32, 12725-12729.

Sweere, A.P.J., Giesselbach, J., Barendse, R., de Krieger, R., Honderd, G., Luyben, K.C.A.M., 1988. Modelling the dynamic behaviour of Saccharomyces cerevisiae and its application in control experiments. Appl. Microbiol. Biotechnol. 28, 116-127.

Takors, R., Wiechert, W., Weuster-Botz, D., 1997. Experimental design for the identification of macrokinetic models and model discrimination. Biotechnol. Bioeng. 56, 564-567.

Theobald, U., Mailinger, W., Baltes, M., Rizzi, M., Reuß, M., 1997. In vivo analysis of metabolic dynamics in Saccharomyces cerevisiae: I. Experimental observations. Biotechnol. Bioeng. 55(2), 305-316.

Thomas, R., 1991. Regulatory networks seen as asynchronous automata: A logical description. J. Theor. Biol. 153, 1-23.

Tomita, M., Hashimoto, K., Takahashi, K., Shimizu, T.S., Matsuzaki, Y., Miyoshi, F., Saito, K., Tanida, S., Yugi, K., Venter, J.C., Hutchison III, C.A., 1999. E-CELL: A software environment for whole-cell simulation. Bioinformatics 15, 1.

Torres, N.V., 1994a. Modeling approach to control of carbohydrate metabolism during citric acid accumulation by Aspergillus niger: I. Model definition and stability of the steady state. Biotechnol. Bioeng. 44, 104-111.

Torres, N.V., 1994b. Modeling approach to control of carbohydrate metabolism during citric acid accumulation of Aspergillus niger: II. Sensitivity analysis. Biotechnol. Bioeng. 44, 112-118. 
Torres, N.V., Voit, E.O., Gonzales-Alcon, C., 1996. Optimization of nonlinear biotechnical processes with linear programming: Application to citric acid production by Aspergillus niger. Biotechnol. Bioeng. 49, 247-258.

Vallino, J.J., 1991. Identification of Branch-Point Restrictions in Microbial Metabolism through Metabolic Flux Analysis and local Network Perturbations. PhD thesis, Massachusetts Institute of Technology.

Vallino, J.J., Stephanopoulos, G., 1993. Metabolic flux distribution in Corynebacterium glutamicum during growth and lysine overproduction. Biotechnol. Bioeng. 41, 633-646.

Vallino, J.J., Stephanopoulos, G., 1994. Carbon flux distributions at the glucose-6-phosphate branch point in Corynebacterium glutamicum during lysine overproduction. Biotechnol. Prog. 10, 327-334.

Van Dam, K., De Koning, W., 1992. A method for the determination of changes of glycolytic metabolites in yeast on a subsecond time scale using extraction at neutral $\mathrm{pH}$. Anal. Biochem. 204, 118-123.

Van Heijden, R.T.J.M., Heijnen, J.J., Hellinga, C., Romein, B., Luyben, K.C.A.M., 1994. Linear constraint relations in biochemical reaction systems: I. Classification of the calculability and the balanceability of conversion rates. Biotechnol. Bioeng. 43, 3-10.

Van Heijden, R.T.J.M., Romein, B., Heijnen, J.J., Hellinga, C., Luyben, K.C.A.M., 1994. Linear constraint relations in biochemical reaction systems: II. Diagnosis and estimation of gross errors. Biotechnol. Bioeng. 43, 11-20.

Varma, A., Palsson, B.O., 1994. Metabolic flux balancing: basic concepts, scientific and practical use. Bio/Technology 12, 994-998.

Varner, J., Ramkrishna, D., 1999. Metabolic engineering from a cybernetic perspective: 1. Theoretical preliminaries. Biotechnol. Prog. 15, 407-425. 
Visser, D., van der Heijden, R., Mauch, K., Reuss, M., Heijnen, S., 2000. Tendency modeling: A new approach to obtain simplified kinetic models of metabolism applied to Saccharomyces cerevisiae. Metabolic Engineering 2. In Press.

Voit, E.O., 1991. Canonical nonlinear modeling: S-system approach to understanding complexity. Van Nostrand Reinhold.

Westerhoff, H.V., van Dam, K., 1987. Mosaic Nonequilibrium Thermodynamics and Control of biological Free-Energy Transduction. Elsevier Amsterdam.

Westhead, D., Clark, D., Murray, C., 1997. A comparison of heuristic search algorithms for molecular docking. J. Comput. Aided Mol. Des. 11, 209-228.

Wiechert, W., de Graaf, A.A., 1996. In vivo stationary flux analysis by ${ }^{13} \mathrm{C}$ labelling experiments. Adv. Biochem. Eng. Biotechnol. 54, 109-154.

Wiechert, W., de Graaf, A.A., 1997. Bidirectional reaction steps in metabolic networks. Part I: Modeling and simulation of carbon isotope labelling experiments. Biotechnol. Bioeng. 55, 101117.

Wiechert, W., Siefke, C., de Graaf, A.A., Marx, A., 1997. Bidirectional reaction steps in metabolic networks. Part II: Flux estimation and statistical analysis. Biotechnol. Bioeng. 55, 118-135.

Wiechert, W., Möllney, M., Isermann, N., Wurzel, M., de Graaf, A.A., 1999. Bidirectional reaction steps in metabolic networks. Part III: Explicit Solution and Analysis of Isotopomer Labelling Systems. Biotechnol. Bioeng., 66(2): 69-85.

Wiechert, W., 2001. ${ }^{13} \mathrm{C}$ Metabolic Flux Analysis. Metabolic Engineering. In Press.

Zupke, C., Stephanopoulos, G., 1994. Modeling of isotope distributions and intracellular fluxes in metabolic networks using atom mapping matrices. Biotechnol. Prog. 10, 489-498. 


\section{Figures and Captions}

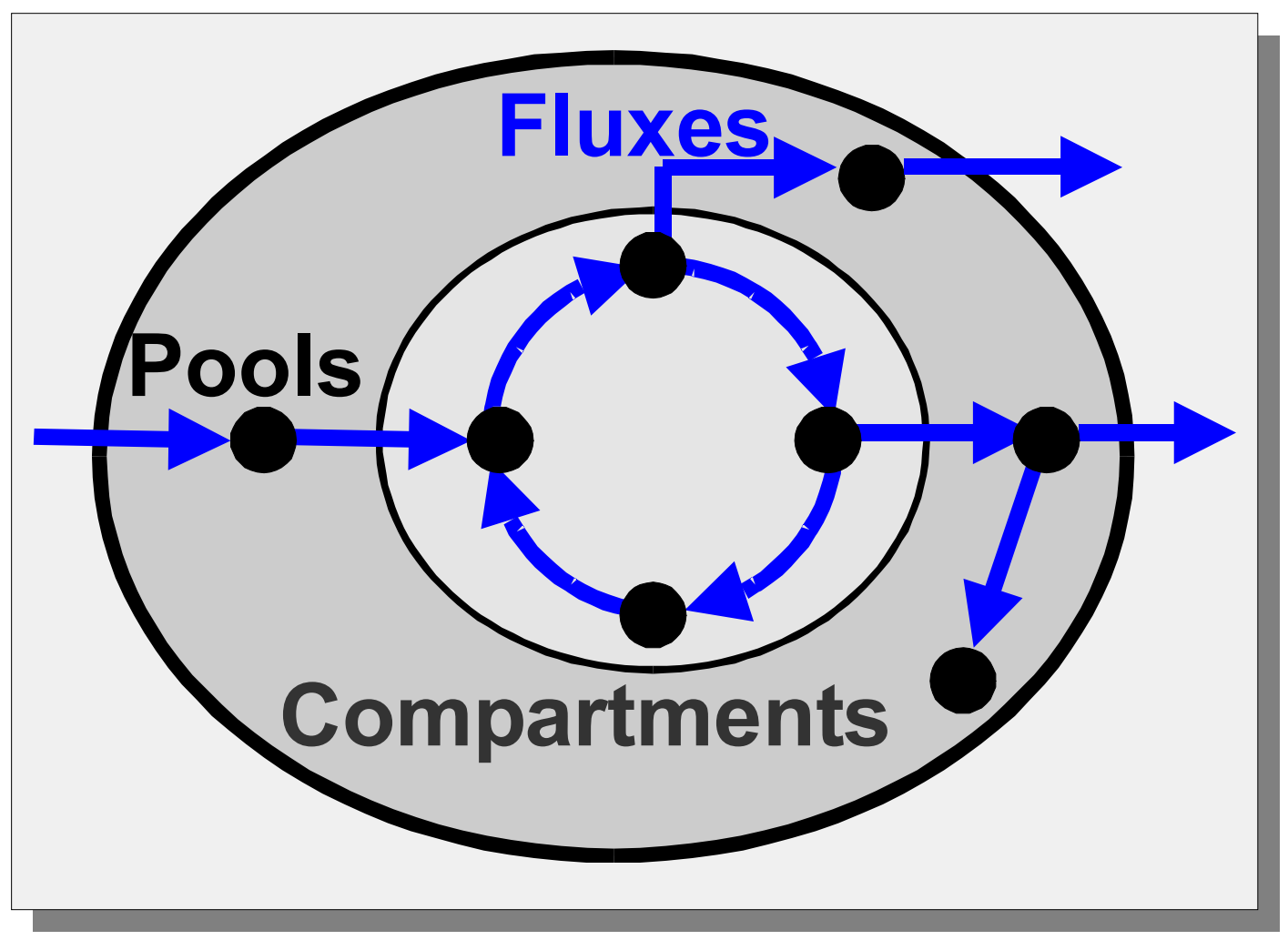

Figure 1 The standard abstraction of typical metabolic models: The cell population is replaced by an "average" single cell. The cell may be compartmented and the substance pools are homogeneously distributed within their respective compartment. Effects of the cell cycle, biological rhythms, cell age and morphology, intracellular concentration gradients or bioreactor inhomogenities are neglected. 


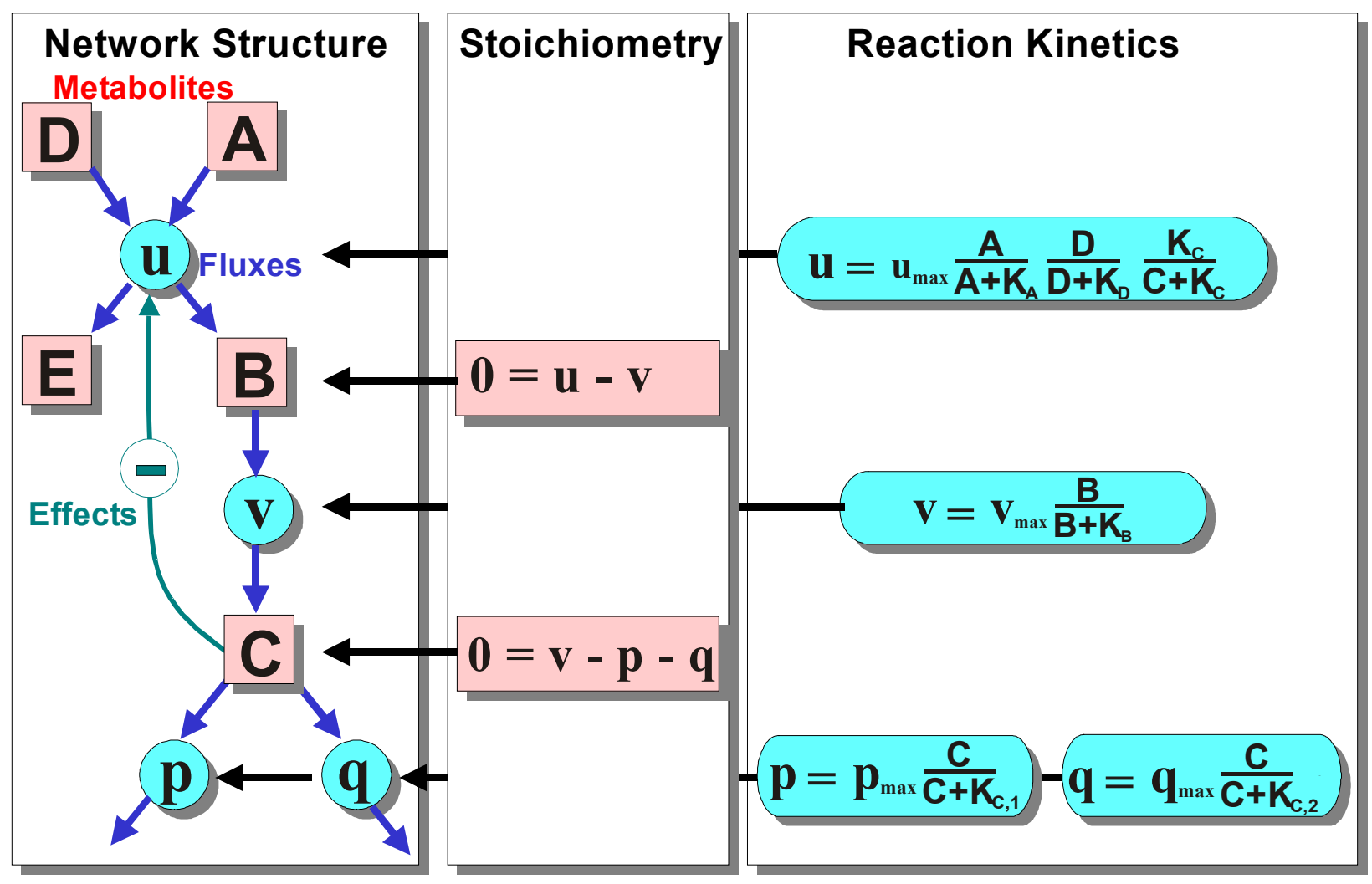

Figure 2: Structural, stoichiometric and kinetic representation of a metabolic system. The structural model specifies all metabolites, reaction steps and regulatory effects. The stoichiometric model adds one linear flux balance equation per intermediate metabolite assuming stationarity. Finally the kinetic model supplies one enzyme kinetic formula for each reaction step. 

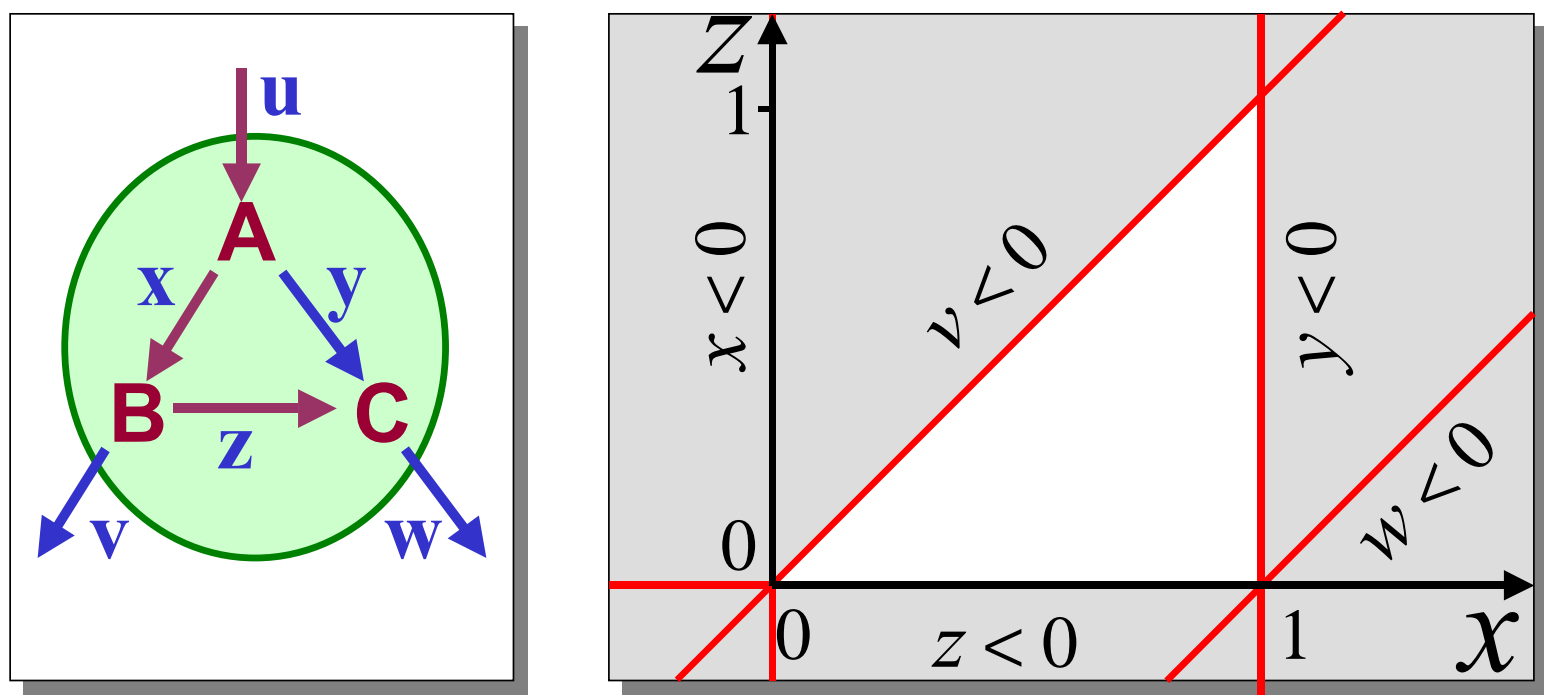

Figure 3: Space of feasible fluxes for a simple reaction network. From the fluxes $u, x, z$ and the stoichiometric relations the other fluxes can be computed as $y=u-x, v=x-z$ and $w=y+z=u-x+z$. By scaling all fluxes relative to the substrate uptake $u$ it can be assumed that $u=1$. If all fluxes are unidirectional the space of feasible fluxes in the $x$-z-plane can be immediately constructed from the relations $x, y, z, v, w>0$. It describes all possible physiological modes in which the system can operate. 


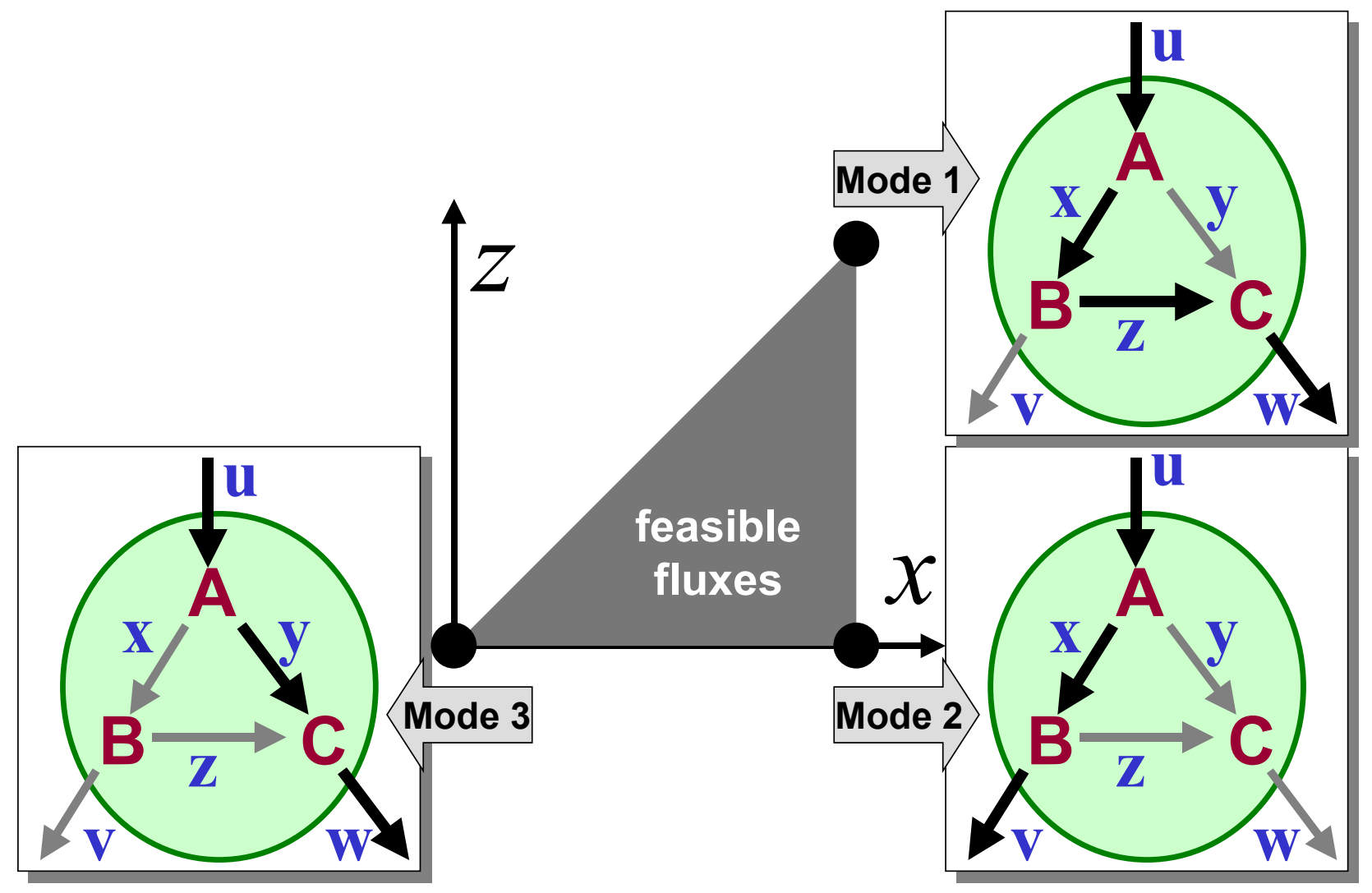

Figure 4: Extremal points of the feasible flux space from Figure 3. To each extremal point corresponds a certain physiological mode of the network where several fluxes vanish. The nonvanishing fluxes constitute a biochemical pathway that can operate autonomously. Alternatively the extremal points can be interpreted as physiological operation modes that minimize or maximize certain fluxes in the network. 

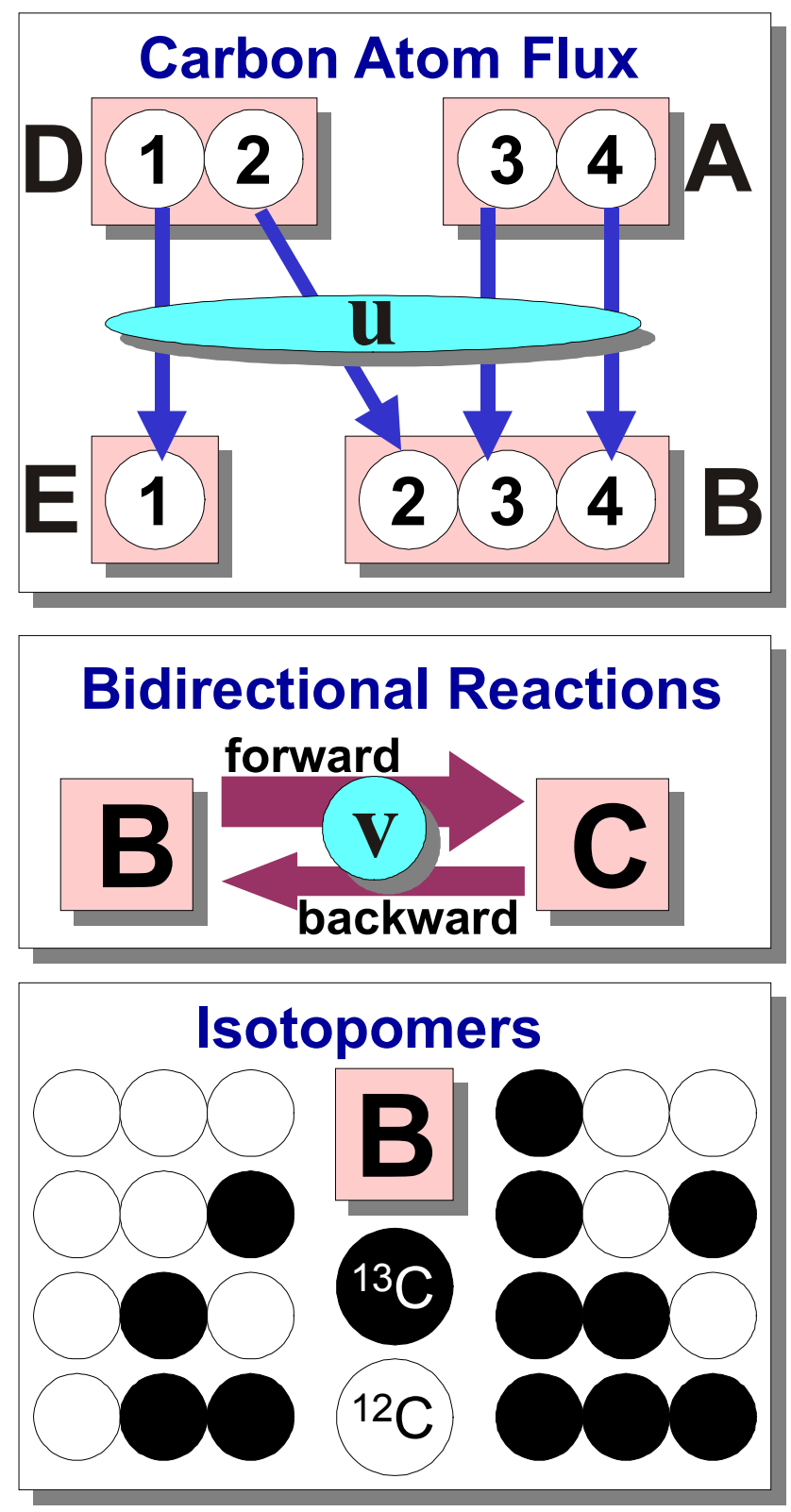

Figure 5: New concepts that distinguish the ${ }^{13} \mathrm{C}$ metabolic flux analysis method from pure stoichiometric flux analysis: i) The carbon atom transitions of each reaction step must be known. In the example the second carbon atom of $\mathrm{D}$ becomes the first carbon atom of $\mathrm{B}$ in the reaction step $u$. ii) The forward and backward reaction of a bidirectional reaction step can be distinguished. iii) Balance equations must be formulated for each isotopomer of all metabolites in the system. The picture shows the 8 isotopomers of the metabolite B which has a carbon skeleton with three carbon atoms. 


\section{Metabolic Network}
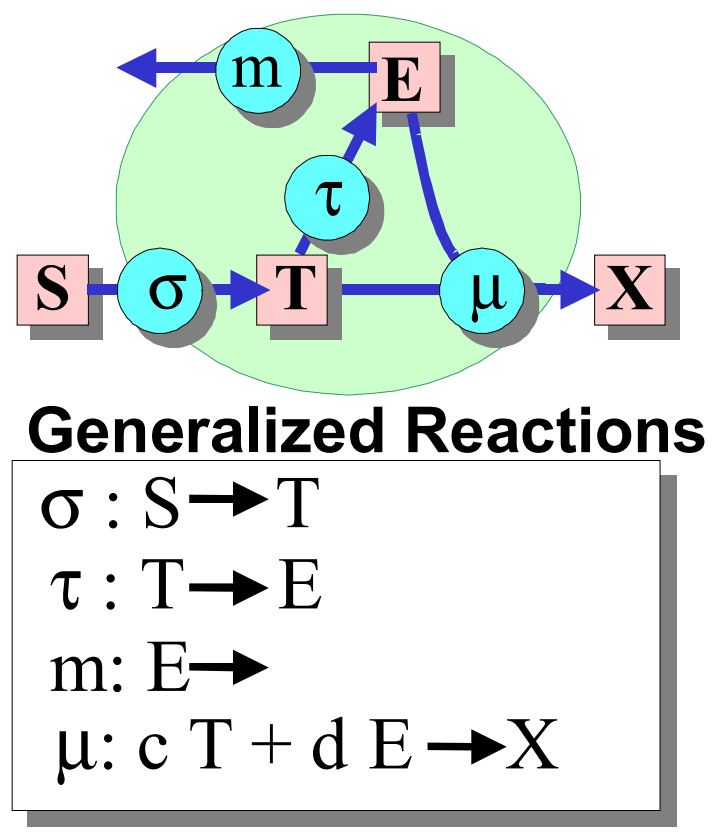

Stoichiometry

$$
\begin{aligned}
& T: \sigma-\tau-c \cdot \mu=0 \\
& E: \tau \quad m \quad \mathrm{~d} \cdot \mu=0 \\
& \text { Kinetics } \\
& \sigma(S)=\sigma_{\max } \cdot S /\left(S+K_{m}\right) \\
& m(S)=m_{0}
\end{aligned}
$$

Figure 6: Derivation of Pirt's classical maintenance model for cellular growth from a generalized reaction network with abstract lumped pools and formal reaction kinetics. Pools: S-substrate, Tintracellular intermediate, E-energy, X-biomass. Fluxes: $\sigma$-substrate uptake, $\mu$-growth rate, $\tau$ energy production, $m$-maintenance. The (abstract) pool units are chosen such that only two generalized stoichiometric coefficients $c, d$ are required. From the stoichiometric relations the growth rate and energy production can be represented as $(c+d) \cdot \mu=\sigma-m,(c+d) \cdot \tau=c \cdot m+d \cdot \sigma$. Filling in formal kinetic terms for $\sigma$ and $m$ completes the classical maintenance model. 


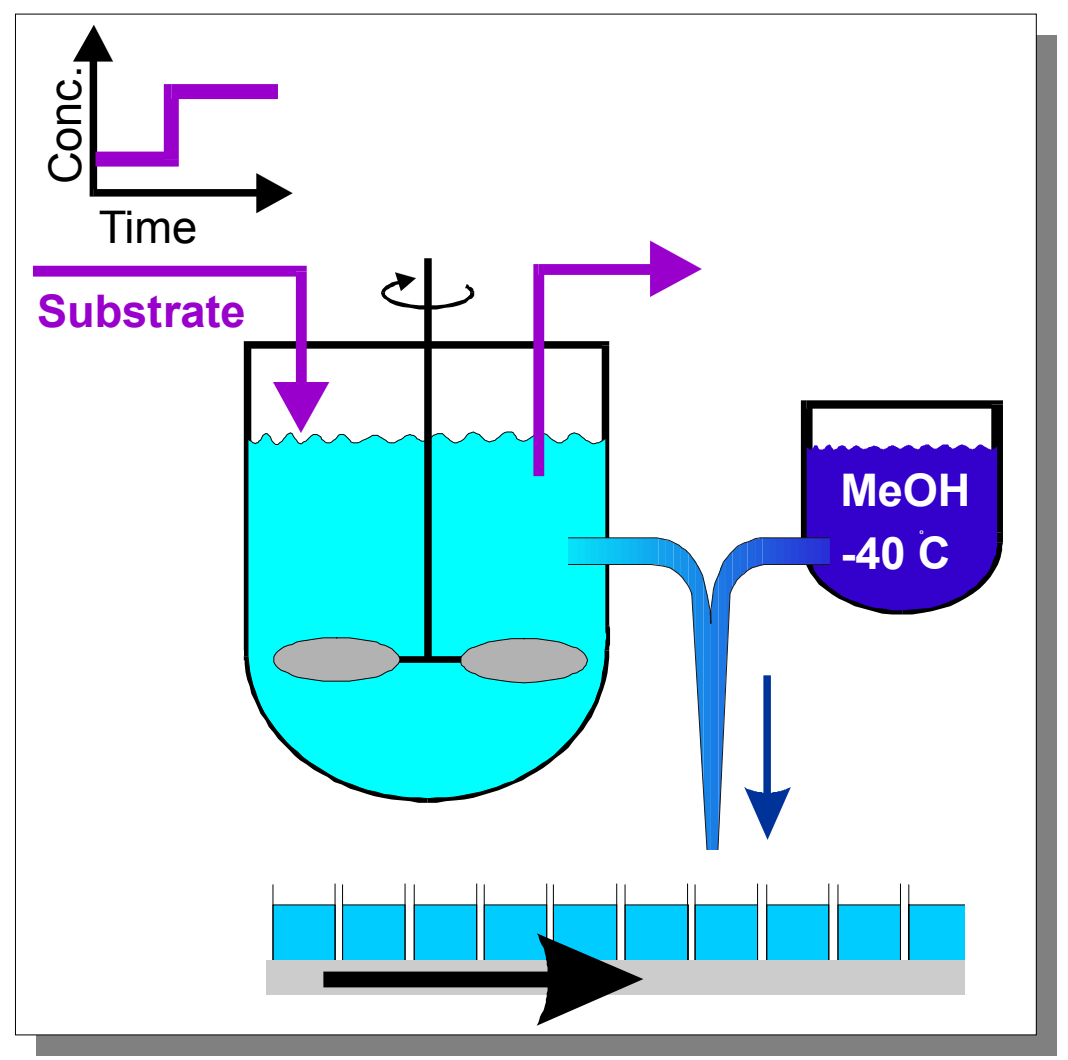

Figure 7: Schematic representation of a rapid sampling experiment. The microorganism is cultivated in a continuously driven bioreactor. A substrate pulse is imposed to stimulate an intracellular metabolic response. Samples are taken in very short time intervals $(5 \mathrm{~Hz})$ and the cell metabolism is stopped by methanol quenching. Afterwards the cells are disrupted and the intracellular pool sizes are measured (Schaefer, 1999). 


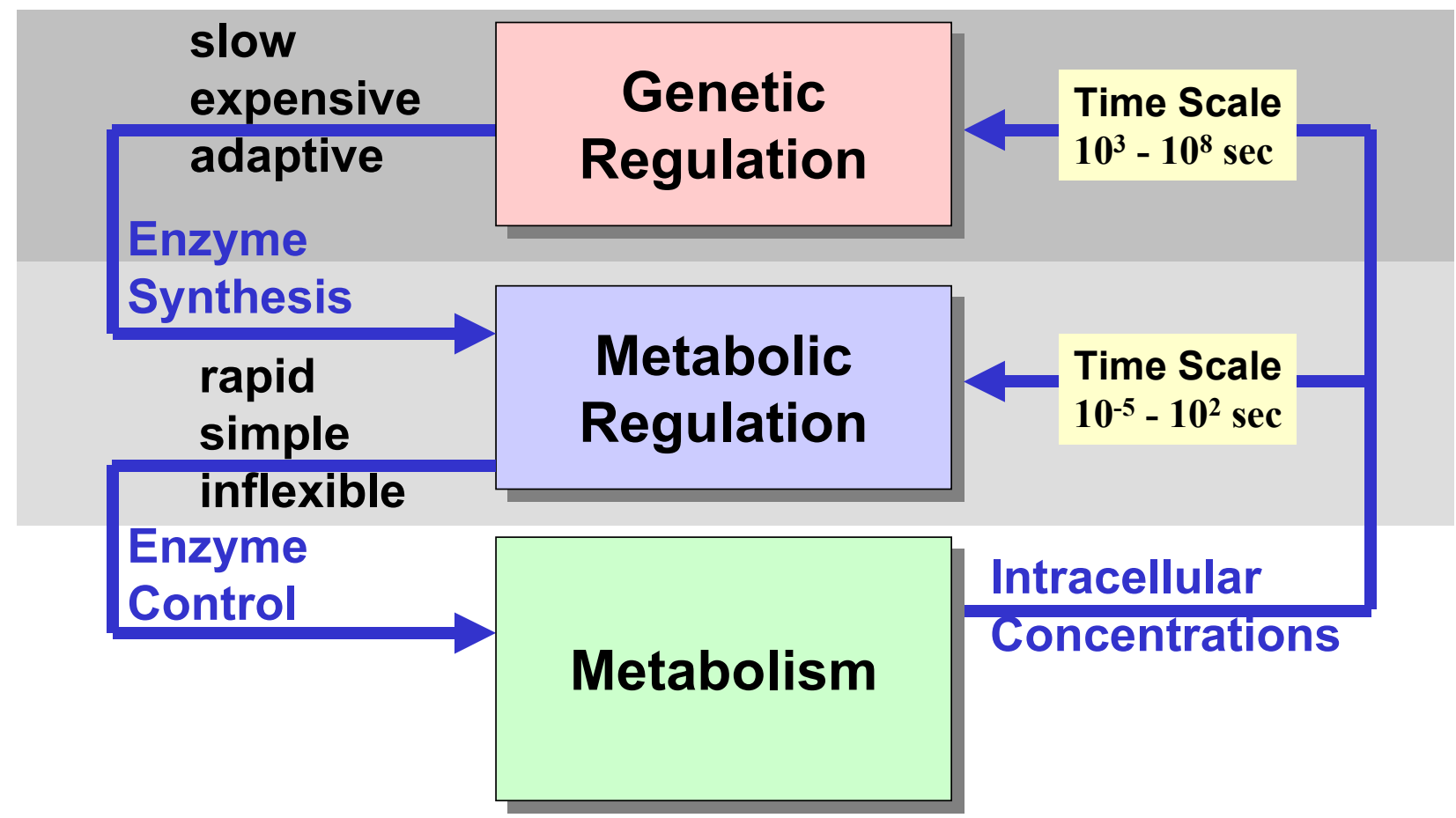

Figure 8: Cascaded structure of the metabolic and genetic regulation networks. While metabolic regulation on the enzyme level takes place at the short time scale, genetic regulation has much larger time constants but is adaptive with respect to changing external conditions. 


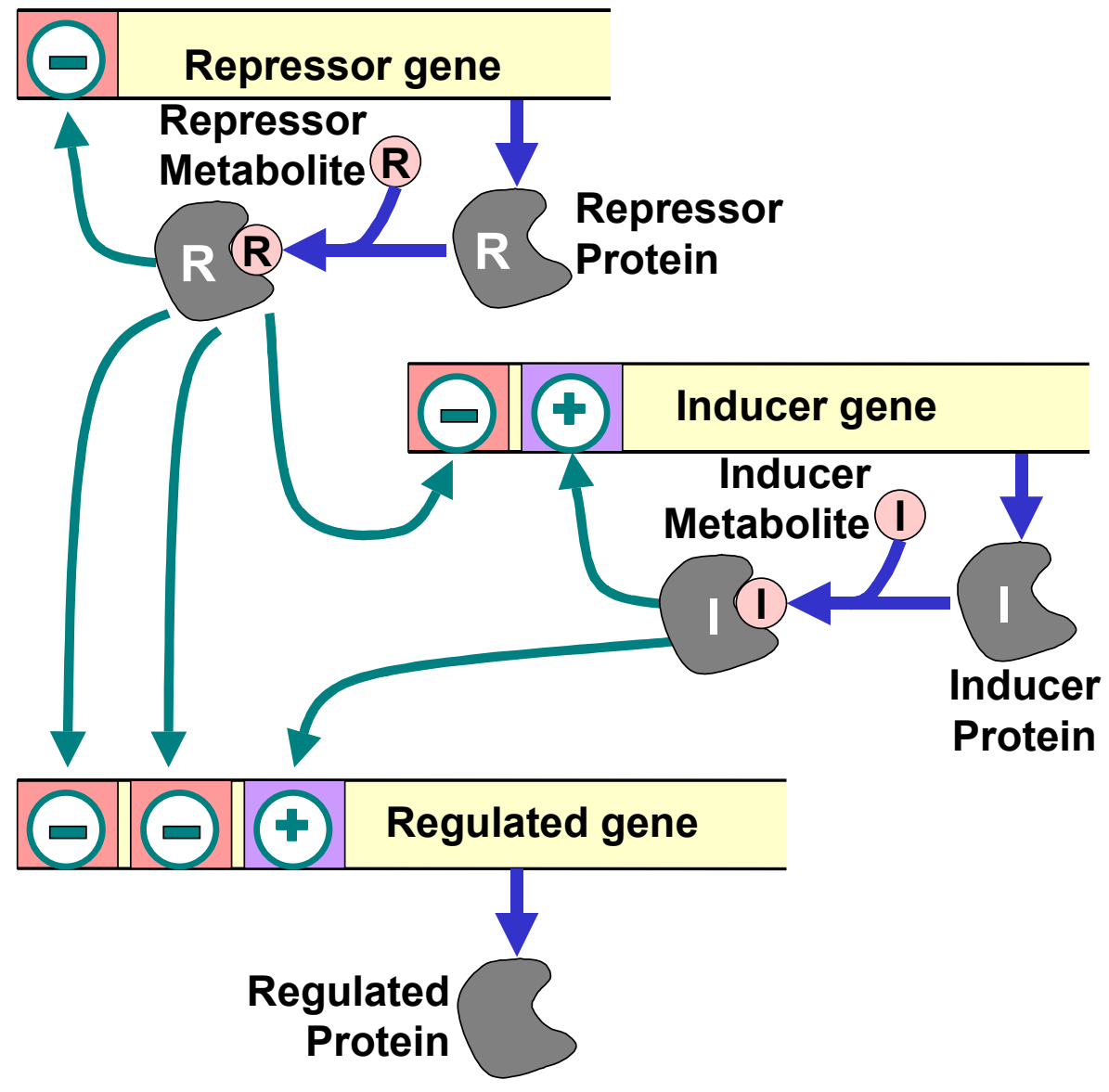

Figure 9: Example of the different binding mechanisms involved in a regulon. The expression of the target protein below is regulated by a repressor and an inducer protein with the corresponding repressor and inducer metabolites. Assuming genes and proteins to be homogeneously distributed substances in the cell population the regulon can be modeled with a network of binding and expression steps. The dynamics of each step may be represented by classical mass action kinetics. Mechanism described in (Agger, Nielsen, 1999). 